\title{
Non-Cooperative Multicast and Facility Location Games
}

\author{
Chandra Chekuri, Julia Chuzhoy, Liane Lewin-Eytan, \\ Joseph (Seffi) Naor and Ariel Orda
}

\begin{abstract}
We consider a multicast game with selfish non-cooperative players. There is a special source node and each player is interested in connecting to the source by making a routing decision that minimizes its payment. The mutual influence of the players is determined by a cost sharing mechanism, which in our case evenly splits the cost of an edge among the players using it. We consider two different models: an integral model, where each player connects to the source by choosing a single path, and a fractional model, where a player is allowed to split the flow it receives from the source between several paths. In both models we explore the overhead incurred in network cost due to the selfish behavior of the users, as well as the computational complexity of finding a Nash equilibrium.

The existence of a Nash equilibrium for the integral model was previously established by the means of a potential function. We prove that finding a Nash equilibrium that minimizes the potential function is NP-hard. We focus on the price of anarchy of a Nash equilibrium resulting from the best-response dynamics of a game course, where the players join the game sequentially. For a game with $n$ players, we establish an upper bound of $O\left(\sqrt{n} \log ^{2} n\right)$ on the price of anarchy, and a lower bound of $\Omega(\log n / \log \log n)$. For the fractional model, we prove the existence of a Nash equilibrium via a potential function and give a polynomial time algorithm for computing an equilibrium that minimizes the potential function. Finally, we consider a weighted extension of the multicast game, and prove that in the fractional model, the game always has a Nash equilibrium.
\end{abstract}

\section{Index Terms}

Multicast game, Nash equilibrium, Price of anarchy, Price of stability.

Chandra Chekuri, Lucent Bell Labs, Murray Hill, NJ 07974, e-mail: chekuri@lucent.com Julia Chuzhoy, School of Mathematics, Institute for Advanced Study, Princeton NJ 08540, email: cjulia@math.ias.edu Liane Lewin-Eytan, Department of Electrical Engineering, Technion, Haifa 32000, Israel, e-mail: liane@tx.technion.ac.il Joseph (Seffi) Naor, Computer Science Department, Technion, Haifa 32000, Israel. Part of this research was done while visiting Microsoft Research, Redmond, WA 98052. This research is supported in part by US-Israel BSF Grant 2002276. e-mail: naor@cs.technion.ac.il Ariel Orda, Department of Electrical Engineering, Technion, Haifa 32000, Israel, e-mail: ariel@ee.technion.ac.il. 


\section{INTRODUCTION}

In many networking scenarios, including the Internet, network users are free to act according to their individual interests, without taking into account overall network performance. Users thus may make selfish decisions (strategy choices) based on the state of the network, which depends (among other factors) on the behavior of other users, resulting in a non-cooperative game. Naturally, these scenarios call for a gametheoretic approach for studying both the behavior of such non-cooperative users, as well as their impact on the network performance. More specifically, we are interested in the properties of Nash equilibrium solutions which are the stable outcomes of a non-cooperative game. We note that there is a considerable amount of research dealing with non-cooperative games in networks [16], [19], [26], [29], [30], [32].

A scenario frequently encountered is the situation where each edge has a load-dependent latency function, and each user aims to minimize the total latency from its source to its destination. In this framework, both simple and general network topologies were studied, as well as various types of latency functions and different constraints on the strategies of the users [16], [26], [29], [30], [32].

While unicast is the traditional form of routing, it results in a waste of resources (e.g., bandwidth) when a source transmits the same data to multiple destinations. Multicast routing schemes provide adequate solutions for such transmissions, and are an important emerging area. An essential question that has been studied extensively in this context is how to distribute the cost of the transmission among the various receivers [1], [5], [9], [12], [15]. However, these works do not consider non-cooperative game scenarios.

We consider a multicast game with selfish non-cooperative players. There is a special source node and each player is interested in connecting to the source by making a routing decision that minimizes its payment. Thus, the strategies of the players in the game correspond to the different paths by which the players can connect to the source. Each player independently chooses a strategy minimizing its payment. The mutual influence of the players is determined by a cost sharing mechanism that stipulates how the cost of each edge in the network is shared among its users. While typical models for non-cooperative games in networks have focused on congestion effects, where a resource utility deteriorates with the number of users that share it, an important class of resource sharing problems occurs when a fixed cost needs to be shared between a set of users. This game was recently introduced by Anshelevich et al. [4]. In this paper we study a natural cost sharing mechanism that falls into the above framework, where the cost of an edge is split evenly among all the players using it. More precisely, if $k$ players use edge $e$ of cost $c_{e}$, then each player pays $c_{e} / k$ for this edge. This cost sharing formula has an intuitive appeal and it was investigated in several studies [9], [12]; it is also the outcome of the Shapley value [31].

Further motivation for the multicast game we consider is provided by the facility location problem, which is of fundamental interest in operations research. In a facility location game, we are given a set of facilities, with an opening cost associated with each facility. Additionally, we have a set of clients, and for each client-facility pair, we are given a cost that the client must pay for connecting to the facility. Each client needs to connect to one facility. A natural cost sharing mechanism for facility location is splitting the opening cost of each facility between the clients served by it. Additionally, each client pays for connecting to the facility serving it. Naturally, the clients seek to minimize their total payment, thus defining a non-cooperative game. This game constitutes a special case of the directed multicast game: given an instance of the facility location game, we add a source, connect each facility to the source with an edge of cost equal to the opening cost of the facility, and then connect each client to each facility with a directed edge of cost equal to the corresponding connection cost.

We consider two different models: an integral model, where each user connects to the source through a single path, and a fractional model, where each user is allowed to split (fractionally) its connection to the source into several paths, i.e., one unit of flow is sent fractionally by the source to the user. The fractional model, in addition to being a relaxation of the integral model, is interesting in its own right, as it is a splittable multicast model which can be implemented via network coding [2], [3], [18]. The games resulting from these models are referred to as the integral multicast game and the fractional multicast game, respectively. 
A crucial property of our multicast game is that the per-user cost share on an edge is non-increasing in the number of users of the edge. Although, in this respect, the game differs from a classic congestion game, the integral multicast game does belong to the well known class of congestion games, that was first defined by Rosenthal [28] and has been widely investigated [13], [23], [25], [31], [33]. Rosenthal showed that a potential function can be defined for each congestion game with the property that the potential decreases if a player makes a move that improves its selfish cost. This shows that every congestion game has a Nash equilibrium. Moreover, there is a one-to-one correspondence between Nash equilibrium solutions and the solutions defining a local minimum of Rosenthal's potential function. Since the integral multicast game belongs to the class of congestion games, it has a Nash equilibrium and a potential function. We note that, for the integral model, the cost sharing mechanism guarantees that a Nash equilibrium induces a tree. The Nash equilibrium of the multicast game raises several natural questions. We focus in this paper on the inefficiency resulting from the selfish behavior of the players, and on the computational complexity of finding a Nash equilibrium.

We quantify the inefficiency resulting from a non-cooperative game through the ratio between the cost of a Nash equilibrium multicast tree and the cost of an optimal Steiner tree spanning the players. In keeping with common terminology [20], [27], this ratio is called the price of anarchy and it quantifies the "penalty" incurred by lack of cooperation (or coordination) between the players in a non-cooperative game.

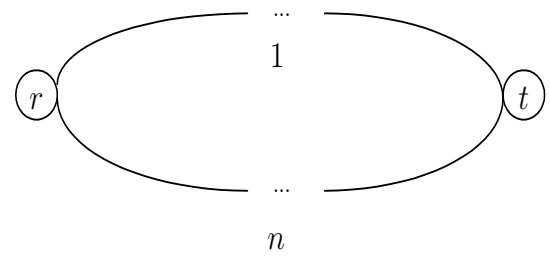

Fig. 1. Cost of a Nash equilibrium tree can be $n$ times the cost of an optimum Steiner tree.

Consider the graph in Figure 1 consisting of a source $r$ and a node $t$ with two parallel paths connecting them. The cost of one path is $n$, while the cost of the other path is 1 . There are $n$ players at $t$ who want to connect to the source $r$. A solution where all players use the expensive path, each paying one unit, is a Nash equilibrium with a cost of $n$. A different and much cheaper Nash equilibrium is the one in which the players use the path of cost 1 . Note that this second equilibrium is also the minimum cost Steiner tree connecting the players to the source. Thus, the price of anarchy for this game can be very large. Notice however that the expensive solution cannot be reached if the players join an initially empty game one-by-one, each of them choosing the cheapest path to connect to the source. In this paper, we investigate the price of anarchy of the integral multicast game for such scenarios. Motivated by the existence of large-cost Nash equilibria, the notion of price of stability was introduced in [4]: it is defined as the ratio between the cost of a Nash equilibrium of minimum cost and the cost of an optimal Steiner tree. In the above example, the price of stability is 1 in contrast to the price of anarchy which is $n$. For directed graphs, it was shown in [4] that the price of stability is $\Theta(\log n)$; for undirected graphs, an upper bound of $O(\log n)$ on the price of stability is known [4], however, no non-trivial lower bounds are known. The price of stability of the multicast game in undirected graph was recently investigated by [10]. It is shown in [10] that for the special case where every vertex is associated with a selfish player, the price of stability is $O(\log \log n)$.

Even if the price of stability in undirected graphs is small, we still have two important questions to answer. Can a Nash equilibrium achieving (or approximating) the price of stability be computed in polynomial time? Second, can a good equilibrium be achieved as a consequence of best-response dynamics? That is, a course of the game where each player, in its turn, makes a routing decision that minimizes its cost. The price of anarchy of such a solution strongly depends on the initial configuration from which the players start. For example, if the starting solution is a Nash equilibrium with a large price 
of anarchy, as in the example in Figure 1, then best-response dynamics would not alter the solution. It is shown in [4] that even in directed graphs, if the initial configuration is a Steiner tree of cost $C$, then the best-response dynamics would lead to a Nash equilibrium of cost at most $O(C \log n)$. This is shown using Rosenthal's potential function, which can only decrease with each best-response move. This is also a constructive proof that the price of stability is $O(\log n)$. In [4], the above argument is used to suggest a mechanism in which a central authority starts the process by first computing a near-optimal Steiner tree on the receivers, and then allows the users to follow their best-response dynamics.

In this paper we take an approach that does not rely on a central trusted authority starting the game in a specific starting configuration. There are several situations in which having such an authority is expensive or infeasible. Further, not all players might be available at the same time. In an online setting, players might arrive one by one to join a multicast service from the source. Motivated by these issues, we explore in this paper the following two-round setting. In the first round, players join the game one by one starting from an "empty" configuration. Upon arrival, each player picks a path selfishly. Once reaching the solution constructed by the players joining one by one, the natural game course induced by best-response dynamics continues in the second round until a Nash equilibrium is reached. We stress that in the second round, unlike the first one, a player may play many times. We assume that the arrival of the players in the first round, as well as the order in which the players play in the second round, is adversarial. Our model is inspired by the round model considered by Mirrokhni and Vetta [24] to analyze convergence issues in competitive games.

Our Results: We focus on undirected graphs. For the integral multicast game, we establish an upper bound of $O\left(\sqrt{n} \log ^{2} n\right)$ on the price of anarchy of the best-response dynamics in the setting where the players join the game sequentially starting from an "empty" configuration. We then present a lower bound of $\Omega\left(\frac{\log n}{\log \log n}\right)$ on the price of anarchy of this game. It is an interesting open question whether a polylogarithmic upper bound can be shown in this setting. We also prove that the problem of computing a Nash equilibrium minimizing Rosenthal's [28] potential function is NP-hard. It remains an open question whether a Nash equilibrium of the integral multicast game can be computed in polynomial time. We note that Fabrikant et al. [8] investigated the complexity of computing a pure Nash equilibrium for the class of congestion games, where the cost of a facility is a non-decreasing function of the number of its users, and showed that it is PLS-complete for general network congestion games. However, their proof heavily depends on the non-decreasing property of the cost sharing mechanism, and therefore does not seem to hold in our model.

For the fractional multicast game, we prove the existence of a Nash equilibrium by extending Rosenthal's potential function. Our main result for this model is that a Nash equilibrium minimizing Rosenthal's potential function can be computed in polynomial time using linear programming. This result should be contrasted with our proof that it is NP-hard to compute an integral Nash equilibrium minimizing Rosenthal's potential function. We observe that the fractional Nash equilibrium minimizing the potential function has a price of anarchy of $O(\log n)$.

The results obtained for the fractional model hold also for more general settings, where the cost sharing mechanisms are cross monotone, which intuitively means that the share of a player on an edge cannot increase when additional players use it. Furthermore, the results also hold in the setting where there are multiple sources and each player needs to connect to at least one source. We note that the fact that our cost sharing mechanism is non-increasing in the number of players using an edge allows us to define a fractional extension. This does not seem possible with a non-decreasing cost sharing mechanism.

Finally, we consider a weighted extension of the multicast game, where each player has a weight, and the cost sharing mechanism splits the cost of an edge among its downstream receivers proportionally to their weights. That is, the cost share of a player for an edge equals the ratio between the player's weight and the total weight of all the players using this edge. For this game we prove that a Nash equilibrium exists in the fractional model. This result should be contrasted with the result of Chen and Roughgarden [6] who showed that a Nash equilibrium does not necessarily exist in the integral weighted multicast game. 


\section{THE MODEL}

We model our network by an undirected graph $G=(V, E)$. Let $c: E \rightarrow \mathcal{R}^{+}$be a non-negative edgecost function, and we denote by $c_{e}$ the cost of edge $e \in E$. There is a special vertex $r \in V$ called root (or source) and a multi-set of $n$ vertices $N=\left\{t_{1}, t_{2}, \ldots, t_{n}\right\}$ representing multicast users (also called players or terminals).

In the integral model, the goal of each user is to choose a single path $P$ connecting it to the root, while minimizing its payment, which consists of the sum of the payments for the edges along $P$. A course of action chosen by player $i$ at any time is called its strategy and is denoted by $s^{i}$. In the integral model, a strategy of player $i$ is a path connecting $t_{i}$ to the root. The strategy space of player $i$ (i.e., the set of all its possible strategies) is denoted by $S^{i}$, and in our integral game, it is the set of all the possible paths between $t_{i}$ and the root. The space of all the possible strategy profiles is denoted by $S$, and it is the Cartesian product of the strategy spaces of all the players, $S=S^{1} \times S^{2} \times \cdots \times S^{n}$. At any given moment, a strategy profile (or a configuration) of the game $s \in S$ is the vector of all the strategies of the players, $s=\left(s^{1}, \ldots, s^{n}\right)$. We use $s^{-i}$ to denote vector $s$ without its $i$ th coordinate, and $\left(s^{-i}, \tilde{s}^{i}\right)$ to denote the strategy profile identical to $s$, except that the $i$ th coordinate is replaced by $\tilde{s}^{i}$. Given a strategy profile $s, c^{i}(s) \equiv c\left(s^{i}\right)$ denotes the payment of player $i$ (the cost of its path $s^{i}$ ), and $n_{e}(s)$ denotes the number of players using edge $e$. Payment of user $i$ for edge $e$ is denoted by $c_{e}^{i}(s)$ and is determined by the cost sharing mechanism. We consider a natural cost sharing mechanism, where the cost of every edge is split evenly between the players sharing it. Thus, the payment of player $i$ for edge $e$ is $c_{e}^{i}(s)=\frac{c_{e}}{n_{e}(s)}$. We denote by $c(s)$ the sum of the costs of the edges participating in $s$ (we say that edge $e$ participates in a strategy profile $s$, if and only if at least one player chooses a path containing $s$ to connect to the source). Let $H(k)$ denote the Harmonic number $\sum_{j=1}^{k} \frac{1}{j}$.

A strategy profile $s \in S$ is at Nash equilibrium if no player has an incentive to change its routing strategy, assuming that the strategies of the other players are fixed. We assume that a player changes its routing choice if and only if it reduces its payment. A change of strategy by any player is called a Nash defection and the corresponding player is called Nash defector. We assume that at each step the acting player chooses a strategy that minimizes the cost of its path, given the strategies of the other players. We therefore say that at each step the strategy of the current player is a best response to the other players' strategies. Thus, at Nash equilibrium, the strategy of each player is the best response to the strategy choices of the other players.

\section{The Integral Multicast Game}

The integral multicast game is a special case of a congestion game, formulated by Rosenthal [28], who defined a potential function to show that every congestion game possesses a Nash equilibrium. A congestion model is denoted by $\Gamma=\left(N, M,\left(S^{i}\right)_{i \in N},\left(\sigma_{j}\right)_{j \in M}\right)$, where $N$ denotes the set of $n$ players $\{1,2, \ldots, n\}$, and $M$ denotes set of facilities $\{1,2, \ldots, m\}$. For each user $i \in N$, $S^{i}$ is the set of its possible strategies, where each $s^{i} \in S^{i}$ is a subset of facilities from $M$. For each facility $j \in M, \sigma_{j} \in \mathbb{R}^{n}$ denotes the payoffs vector of $j$, where $\sigma_{j}(k)$ is the payoff of each player for using facility $j$ in case exactly $k$ players use $j$. Finally, $x_{j}(s)$ denotes the number of players using facility $j$ according to their strategies defined by the strategy profile $s \in S$. Given a strategy profile $s$ and a facility $j$, we say that $j \in s$ iff $x_{j}(s)>0$.

Rosenthal [28] showed that every congestion game possesses a Nash equilibrium by constructing an appropriate potential function. Later, Monderer and Shapley [25] characterized the class of finite potential games, showing that they coincide with the class of congestion games, where each step performed by a player improving its payoff also decreases (or increases) the value of a global potential function $\Phi$ on the strategy space. Consequently, if $\Phi$ admits a minimal (maximal) value in $S$, then the respective game possesses a (pure-strategy) equilibrium.

The potential function $\Phi(s)$ defined by Rosenthal for the congestion model is the following: 


$$
\Phi(s)=\sum_{j \in s}\left(\sum_{k=1}^{x_{j}(s)} \sigma_{j}(k)\right) .
$$

For completeness, we sketch the proof of the following theorem, presented in [28].

Theorem 1 (Rosenthal [28]): Every congestion game has a pure Nash equilibrium.

Proof: The function $\Phi$ is an exact potential for any congestion game. That is, for every $i \in N$, and every pair of strategy profiles $\left(s^{-i}, s_{1}^{i}\right)$ and $\left(s^{-i}, s_{2}^{i}\right)$, if $c^{i}\left(s^{-i}, s_{1}^{i}\right)<c^{i}\left(s^{-i}, s_{2}^{i}\right)$, then

$$
c^{i}\left(s^{-i}, s_{2}^{i}\right)-c^{i}\left(s^{-i}, s_{1}^{i}\right)=\Phi\left(s^{-i}, s_{2}^{i}\right)-\Phi\left(s^{-i}, s_{1}^{i}\right)
$$

In other words, the decrease in the value of the potential function following a Nash defection is equal to the decrease in the payoff of the respective Nash defector.

For our multicast game, given a strategy profile $s$, the potential function $\Phi$ of [28] is

$$
\Phi(s)=\sum_{e}\left(\sum_{k=1}^{n_{e}(s)} \frac{c_{e}}{k}\right) .
$$

It is easy to see that for every instance of our game, a Nash equilibrium solution is a tree rooted at $r$ spanning $N$.

We now analyze the price of anarchy of a multicast game in an undirected graph. We are interested in a Nash equilibrium that is a consequence of best-response dynamics, where each Nash defector, in its turn, chooses a path to the source minimizing its payment. Finding such a path can be done in polynomial time by using a standard shortest path algorithm. Initially, the players join the game one by one starting from an "empty" configuration and picking a path to the root that minimizes their payment. Once all players are connected to the root, they continue playing until reaching Nash equilibrium. Note that we assume that the order by which the players play is adversarial. In Section III-A we establish an upper bound of $O\left(\sqrt{n} \log ^{2} n\right)$ on the price of anarchy for this game course, and in Section III-B we prove a lower bound of $\Omega\left(\frac{\log n}{\log \log n}\right)$ on the price of anarchy. We also prove that finding a Nash equilibrium minimizing Rosenthal's [28] potential function is NP-hard in Section III-C.

\section{A. Upper Bound}

In this section we establish an upper bound of $O\left(\sqrt{n} \log ^{2} n\right)$ on the price of anarchy of a Nash equilibrium obtained from best-response dynamics. Our analysis is performed in two steps. We first analyze (in Section III-A.1) the first round of the game in which the players connect one-by-one to the root via a cheapest path. The first round finishes when all players are connected to the root. However, the configuration reached by the players after the first round is not necessarily a Nash equilibrium. In the second round, we start from the solution obtained in the first round and follow the natural game course until a Nash equilibrium is reached. In order to bound the price of anarchy of the strategy profile $T$ obtained from the first round, we define the notion of a level tree that serves as a basis of reference for proving the upper bound.

A greedy online Steiner tree [14] is defined as follows. It is the tree obtained when terminals arrive online one by one. Upon arrival, each terminal connects via a cheapest path to the root, where the path cost only consist of the costs of the edges that do not currently belong to the tree. Thus, the $i$ th terminal connects by a cheapest path to the tree induced by the choices of terminals $1, \ldots, i-1$. The total cost of the greedy online Steiner tree is known to be at most a factor of $O(\log n)$ away from the cost of an optimal Steiner tree [14]. We consider the greedy online Steiner tree obtained from the same sequence of arrivals as in the first round of the game. Our goal is to prove that the cost of the solution obtained by the selfish moves of the players is related to the cost of the online Steiner tree. We are, however, unable to show this directly. We overcome this difficulty by first transforming the online Steiner tree to a level 
tree with reduced height using a procedure due to Zelikovsky [34]. The height reduction increases the cost, but maintains ancestor relationships that are critical for the analysis. We prove that the cost of the solution obtained from the first round is at most $O(\sqrt{n} \log n)$ times the cost of an optimal Steiner tree.

We complete our analysis in Section III-A.2. Starting from the solution obtained from the first round, we follow the natural game course until a Nash equilibrium is reached. The Nash defections performed in the second round can only decrease the potential function value, and thus we lose at most another factor of $O(\log n)$ with respect to the cost of the solution obtained from the first round.

We paraphrase below the height reduction lemma of Zelikovsky that we need. A bound claimed in [34] proved to be incorrect and a weaker correct bound is established in [11].

Lemma 3.1: Let $T=(V, A)$ be an in-tree rooted at $r \in V$ and let $c: A \rightarrow \mathcal{R}^{+}$be a non-negative cost function on $A$. Let $G=\left(V, A_{G}\right)$ be the transitive closure of $T$ and let $c^{\prime}: A_{G} \rightarrow \mathcal{R}^{+}$be such that $c^{\prime}(u, v)$ is the shortest $c$-path from $u$ to $v$ in $T$. Then, given integer $h>1$, there exists an in-tree tree $T^{\prime}=\left(V, A^{\prime}\right)$ in $G$, where $A^{\prime} \subseteq A_{G}$, of height at most $h$ such that $\sum_{a \in A^{\prime}} c^{\prime}(a) \leq h \cdot|V|^{1 / h} \sum_{a \in A} c(a)$.

1) The First Round: We begin by analyzing the first round of the game in which players arrive one by one and pick a path selfishly. Let the sequence of arrivals of the terminals be $t_{1}, t_{2}, \ldots, t_{n}$ (renumber if necessary), and let $T$ be the resulting solution. We assume that the players start from an empty configuration. Note that $T$ need not be a tree.

Definition 1: A level tree $T^{\prime}$ on the vertex set $\left\{r\left(=t_{0}\right), t_{1}, t_{2}, \ldots, t_{n}\right\}$, with a cost function $d: E \rightarrow \mathbb{R}$, is defined to be a tree having the following properties for each terminal $t_{i}$. (i) For $1 \leq i \leq n$, the ancestor of terminal $t_{i}$ in $T^{\prime}$ belong to $t_{0}, t_{1}, t_{2}, \ldots, t_{i-1}$, i.e., terminals that have arrived before $t_{i}$. (ii) Let $t$ and $t_{i}$ be two terminals in $T^{\prime}$, such that $t$ is the parent of $t_{i}$. Then the cost of the edge $\left(t_{i}, t\right)$ in $T^{\prime}$, denoted by $d(i)$, is no less than the cost of the cheapest path between $t_{i}$ and $t$ in $G$.

Define $c\left(T^{\prime}\right)=\sum_{i=1}^{n} d(i)$. Let $T(i)$ denote the state of $T$ after the arrival of $t_{1}, \ldots, t_{i}$. Let $P_{i}$ denote the path chosen by $t_{i}$ to the root $r$ in $T$. We denote by $B(i)$ the set of new edges that are added to $T$ when $t_{i}$ joins $T(i-1)$. Let $b(i)=\sum_{e \in B(i)} c_{e}$. Clearly, $c(T)=\sum_{i=1}^{n} b(i)$. Let $c(i)$ be the cost paid by $t_{i}$ when it joins $T$. Clearly, $c(i) \geq b(i)$. Note that in the single round case the cost paid by a player can only decrease during the round.

Given an edge $e$, let $n_{e}(i)$ denote the number of paths (terminals) using $e$ in $T(i)$. We use $c_{e}(i)$ to denote the cost of $e$ as seen by a selfish player in $T(i)$, i.e., $c_{e} / n_{e}(i)$. We use $c_{e}^{+}(i)$ to denote $c_{e} /\left(n_{e}(i)+1\right)$ which is the cost per player for using edge $e$ if an additional player were to use $e$ in $T(i)$. We define $c^{+}(i)$ to be $\sum_{e \in P_{i}} c_{e}^{+}(i)$. The following is immediate.

Fact 3.1: $c^{+}(i) \leq(c(i)-b(i))+b(i) / 2=c(i)-b(i) / 2$.

The edge set of $T$ is partitioned by the sets $B(i), 1 \leq i \leq n$. We now show how we charge the cost of edges in $B(i)$ to $d(1), \ldots, d(i)$. Assume that we are given a level tree $T^{\prime}$ rooted at $r$ having height 2 . Let $t_{i_{1}}, \ldots, t_{i_{m}}$ be the first level terminals, i.e., the children of $r$ in $T^{\prime}$. The second level terminals are the children of $t_{i_{1}}, \ldots, t_{i_{m}}$, i.e., leaves of $T^{\prime}$. Denote by $A\left(t_{i_{j}}\right)$ the children of first level terminal $t_{i_{j}}$.

We first analyze the cost of the edges added to $T$ by the first level terminals.

Lemma 3.2: For the first level terminals,

$$
\sum_{j=1}^{m} b\left(i_{j}\right) \leq \sum_{j=1}^{m} c\left(i_{j}\right) \leq \sum_{j=1}^{m} d\left(i_{j}\right) .
$$

Proof: By Definition 1, for each first level terminal $t_{i_{j}}, 1 \leq j \leq m$, there is a path to the root $r$ of cost at most $d\left(i_{j}\right)$ (without taking into account cost sharing). Therefore, $b\left(i_{j}\right) \leq c\left(i_{j}\right) \leq d\left(i_{j}\right)$.

We now analyze the cost of the edges added to $T$ by the second level terminals.

Lemma 3.3: Let $t_{j}$ be a first-level terminal with children $t_{j_{1}}, t_{j_{2}}, \ldots, t_{j_{k}}$ in $T^{\prime}$. Then

$$
\sum_{i=1}^{k} b\left(j_{i}\right) \leq 2 c^{+}(j)+4 \sum_{i=1}^{k} d\left(j_{i}\right) .
$$


Proof: Assume w.l.o.g. that the arrival order is $t_{j_{1}}, t_{j_{2}}, \ldots, t_{j_{k}}$. Consider what happens when $t_{j_{1}}$ arrives: it can connect to $t_{j}$, and then connect to the root via the path connecting $t_{j}$ to the root. Hence, $c\left(j_{1}\right) \leq d\left(j_{1}\right)+c^{+}(j)$. Now consider terminal $t_{j_{i}}$ for $i>1$. It can connect to $t_{j_{i-1}}$ (paying at most $d\left(j_{i-1}\right)+$ $\left.d\left(j_{i}\right)\right)$, and then follow $P_{j_{i-1}}$ to the root. Hence, the cost of this path is at most $d\left(j_{i-1}\right)+d\left(j_{i}\right)+c^{+}\left(j_{i-1}\right)$, which by Fact 3.1 is at most $d\left(j_{i-1}\right)+d\left(j_{i}\right)+c\left(j_{i-1}\right)-b\left(j_{i-1}\right) / 2$. Thus, we have for $1<i \leq k$,

$$
c\left(j_{i}\right) \leq d\left(j_{i-1}\right)+d\left(j_{i}\right)+c\left(j_{i-1}\right)-b\left(j_{i-1}\right) / 2 .
$$

Adding up the above inequalities, we obtain:

$$
\begin{aligned}
& c\left(j_{k}\right)+\frac{1}{2}\left(b\left(j_{1}\right)+b\left(j_{2}\right)+\ldots+b\left(j_{k-1}\right)\right) \leq \\
& c^{+}(j)+d\left(j_{k}\right)+2\left(d\left(j_{1}\right)+d\left(j_{2}\right)+\ldots d\left(j_{k-1}\right)\right) .
\end{aligned}
$$

Since $b\left(j_{k}\right) / 2 \leq b\left(j_{k}\right) \leq c\left(j_{k}\right)$, we obtain the desired inequality:

$$
\sum_{i=1}^{k} b\left(j_{i}\right) \leq 2 c^{+}(j)+4 \sum_{i=1}^{k} d\left(j_{i}\right) .
$$

We conclude with the next theorem.

Theorem 2: $c(T) \leq 4 c\left(T^{\prime}\right)$.

Proof: We combine Lemmas 3.2 and 3.3 and get:

$$
\begin{aligned}
& c(T)=\sum_{i=1}^{n} b(i)=\sum_{j=1}^{m}\left(b\left(i_{j}\right)+\sum_{t_{\ell} \in A\left(t_{i_{j}}\right)} b(\ell)\right) \\
& \leq \sum_{j=1}^{m} b\left(i_{j}\right)+\sum_{j=1}^{m}\left(2 c^{+}\left(i_{j}\right)+\sum_{t_{\ell} \in A\left(t_{i_{j}}\right)} 4 d(\ell)\right) \\
& \leq \sum_{j=1}^{m} d\left(i_{j}\right)+\sum_{j=1}^{m}\left(2 d\left(i_{j}\right)+\sum_{t_{\ell} \in A\left(t_{i_{j}}\right)} 4 d(\ell)\right) \\
& \leq 4 \sum_{i=1}^{n} d(i) \leq 4 c\left(T^{\prime}\right) .
\end{aligned}
$$

An interesting question is whether the use of level trees which have depth greater than two can lead to better bounds on the price of anarchy. The difficulty with this approach is that for trees with more than two levels, a recursive use of Lemma 3.3 is necessary. However, the recursion introduces extra charges, and it is not clear how to bound them.

2) Completing the Analysis: We first generate the level tree $T^{\prime}$. Note that the greedy online Steiner tree obtained from the sequence of arrivals of the first round of the game has all the properties required by a level tree. The difficulty is that the height of the greedy online Steiner tree can be $\Omega(n)$. We generate a new level tree $T^{\prime}$ from the greedy online Steiner tree by applying Lemma 3.1. The transformation preserves ancestral relationship and thus $T^{\prime}$ remains a level tree, while allowing us to restrict the height of the tree to be $h$ at the expense of increasing its cost by a factor of $h \cdot n^{1 / h}$. By choosing $h=2$, we get a two level tree $T^{\prime}$ and cost at most $2 \sqrt{n}$ times the cost of the greedy online Steiner tree. As the cost of a greedy online Steiner tree is within a factor of $O(\log n)$ away from the cost of an optimal Steiner tree, we get that $c\left(T^{\prime}\right)=O(\sqrt{n} \log n) \cdot c\left(T^{*}\right)$, where $T^{*}$ is an optimal Steiner tree. Therefore, by Theorem 2, $c(T)=O(\sqrt{n} \log n) \cdot c\left(T^{*}\right)$. 
Finally, after reaching the solution $T$ constructed by the terminals in the first round, the natural best response dynamics are followed in the second round until a Nash equilibrium is reached. It is easy to see that the value of the potential function of any configuration is at most $\log n$ times the total cost of the edges used in this configuration. Therefore, the potential function value of $T$ is within at most a factor of $O(\log n)$ away from $c(T)$. The potential function value can only decrease throughout the second round. As the value of the potential function of a solution is always an upper bound on the cost of the edges participating in the solution, we get that the price of anarchy of our game is $O\left(\sqrt{n} \log ^{2} n\right)$.

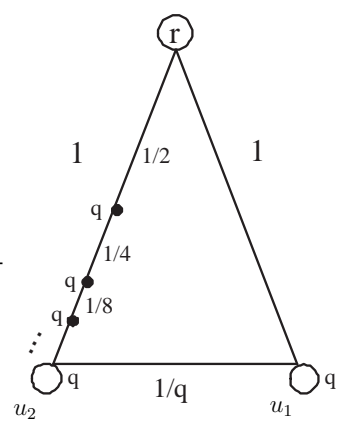

(a)

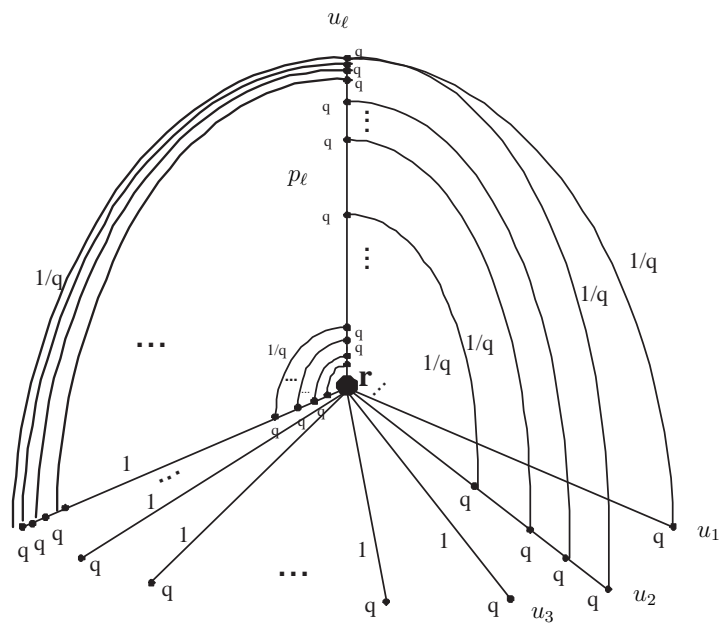

(b)

Fig. 2. (a) Price of anarchy of $2-\epsilon$. (b) Price of anarchy of $\Omega\left(\frac{\log n}{\log \log n}\right)$.

\section{B. Lower Bound}

In this section we present an undirected instance in which best-response dynamics converges to a Nash equilibrium with price of anarchy of $\Omega\left(\frac{\log n}{\log \log n}\right)$. We start with a simple example whose price of anarchy is at least $2-\epsilon$. After that we show how to extend the ideas used in this example to show an $\Omega(\log n / \log \log n)$ lower bound on the price of anarchy.

Let $q$ be a large integer. Our first example is constructed as follows. We start from a root vertex $r$ and additional vertex $u_{1}$ connected to the root by a unit-length edge. We now add another unit-length path from the root to a new vertex $u_{2}$. The edges and the vertices of this path are as follows. Apart from $r$ and $u_{2}$, there are $\log q$ vertices $v_{1}, \ldots, v_{\log q}$ that are placed between $r$ and $u_{2}$ in this order, with vertex $v_{1}$ adjacent to $r$. The distance between $v_{1}$ and $r$ is $\frac{1}{2}$, and for every $i>1$, the distance between $v_{i}$ and $v_{i-1}$ is $2^{-i}$, thus the distance between $v_{\log q}$ and $u_{2}$ is less than $\frac{1}{q}$. Finally, there is an edge of length $\frac{1}{q}$ between $u_{1}$ and $u_{2}$.

The idea is as follows. The first $q$ players joining the game are placed on vertex $u_{1}$. They connect to $r$ via the unit-length edge $\left(r, u_{1}\right)$ and pay $\frac{1}{q}$ each. In the next step we place $q$ players on vertex $v_{1}$. Naturally, they prefer to connect to $r$ via edge $\left(v_{1}, r\right)$ whose cost is $\frac{1}{2}$, instead of connecting via $u_{2}$ and $u_{1}$. Now the cost of the edge $\left(v_{1}, r\right)$ becomes $\frac{1}{2 q}$. When we place the next $q$ players on vertex $v_{2}$, they connect via $\left(v_{2}, v_{1}, r\right)$, as the cost of this path is less than $\frac{1}{4}+\frac{1}{2 q}$ while connecting via $u_{2}$ and $u_{1}$ costs more than $\frac{1}{4}+\frac{1}{q}$. We continue in the same way, placing $q$ users on vertices $v_{3}, \ldots, v_{\log q}$, where the users placed on vertex $v_{i}$ all connect via path $\left(v_{i}, v_{i-1}, \ldots, v_{1}, r\right)$. Finally, we place $q$ players on vertex $u_{2}$, who also prefer to connect via path $\left(u_{2}, r\right)$, as its cost is less than $\frac{1}{q}$. It is easy to see that this configuration is a Nash equilibrium. The cost of this solution is 2 , while the cost of the optimal solution is $1+\frac{1}{q}$, which 
is achieved by connecting all the players on path $\left(u_{2}, r\right)$ via this path and connecting all the players on vertex $u_{1}$ via $u_{2}$. See Figure III-A.2(a) for the resulting instance.

We now show how to generalize the above construction to obtain the bound of $\Omega\left(\frac{\log n}{\log \log n}\right)$ on the price of anarchy. The basic gadget we use in our construction is a log-division of an edge.

Definition 2: Suppose we have an edge $(a, b)$ of length $c$. A log-division of this edge is performed by converting this edge into a path $a, v_{1}, v_{2}, \ldots, v_{\log q}, b$ of the same length. The length of the edge $\left(a, v_{1}\right)$ is $\frac{c}{2}$, and for each $i>1$, the length of edge $v_{i}, v_{i-1}$ is $\frac{c}{2^{i}}$. Thus, the length of edge $\left(v_{\log q}, b\right)$ is less than $\frac{c}{q}$.

A building block of our construction is a path $p$ defined below. We use $\ell=\Omega\left(\frac{\log q}{\log \log q}\right) \operatorname{copies}$ of $p$. The construction of path $p$ is as follows. We start from an edge $(v, u)$ of length 1 . Vertex $u$ is called a level-1 vertex. We now perform $\ell$ iterations. In each iteration, we perform a log-division of every edge $e=\left(w, w^{\prime}\right)$ on path $(v, u)$. When doing this division, the endpoint of $e$ that is closer to $v$ on the path (say, $w)$ serves as $a$ and the other endpoint $\left(w^{\prime}\right)$ serves as $b$. For each iteration $i$, we call the vertices added to the path in this iteration "level $i$ vertices". In our construction, we use $\ell$ copies of path $p$, denoted by $p_{1}, p_{2}, \ldots, p_{\ell}$. The endpoints $v$ of these paths are merged together and form the root $r$. The other endpoints of the paths are denoted by $u_{1}, u_{2}, \ldots, u_{\ell}$. Finally, for each $i, 1 \leq i<\ell$, we connect each of the level- $1,2, \ldots, i$ vertices on path $p_{i}$ to the corresponding vertex on path $p_{\ell}$ by an edge of length $\frac{2}{q}$.

The players are added to the game as follows. First we add $q$ players on vertex $u_{1}$. They connect via path $p_{1}$ to the root. Then, we add players on paths $p_{2}, \ldots, p_{\ell}$ in this order. For $i>1$, we add players on all the vertices of levels $1,2, \ldots, i$ belonging to path $p_{i}$, as well as on vertex $u_{i}$, in the order by which the vertices appear on the path starting from the root. See Figure III-A.2(b) for the resulting instance.

Claim 1: For each $i: 1 \leq i \leq \ell$, for each player $\alpha$ on path $p_{i}$, $\alpha$ connects to the root $r$ via the subpath $(x, r)$ of path $p_{i}$, where $x$ is the vertex on which $\alpha$ lies.

Proof: The proof is by induction on the order in which the players are being added. Let $\alpha$ be some player lying on vertex $x$ of path $p_{i}$.

If $x$ is the first vertex on path $p_{i}$, then it is a level- $i$ vertex. Let $z$ be its closest level- $i-1$ vertex. Player $\alpha$ has two options to connect to the root. One is via the path segment $(x, r)$, and the other is via the path segment $(x, z)$, and then use the edge connecting $z$ to $p_{\ell}$ whose cost is $2 / q$ (all other options are clearly more expensive). However, since the costs of $(x, r)$ and $(x, z)$ are the same, the cheaper option is the path $(x, r)$.

Assume now that $x$ is not the first vertex on path $p_{i}$. Let $y$ be the vertex lying next to $x$ on the sub-path of $p_{i}$ connecting $x$ to $r$. Assume first that $x$ is a level- $i$ vertex. By the induction hypothesis, all the players that were already added to path $p_{i}$ chose to connect to $r$ via this path. Hence the cost of the segment $(y, r)$ is currently at most $1 / q$. Thus, connecting to $r$ via the path $(x \rightarrow y \rightarrow r)$ costs at most $1 / q$ plus the cost of $(x, y)$. The other possibility is to connect to $z$ (the closest vertex of levels $1, \ldots, i-1$ lying on path $p_{i}$ but not on the segment $(x, r)$ of this path), and then use the $2 / q$-cost edge connecting $z$ to $p_{l}$. Since the cost of $(x, z)$ is the same as the cost of $(x, y)$, this is more expensive than connecting via the $(x, r)$ segment of $p_{i}$.

Finally, assume that $x$ is a vertex of $p_{i}$ that belongs to one of the levels $1, \ldots, i-1$. Again, using the induction hypothesis, all the players already added to path $p_{i}$ connect via the path $p_{i}$ to the root. Since the distance from $x$ to the next level- $i$ vertex on the path $(x, r)$ is less than $1 / q$, it means that the cost of connecting $x$ to $r$ via the sub-path $(x, r)$ of $p_{i}$ is less than $2 / q$. The other option would be connecting to path $p_{\ell}$ using a cost $2 / q$ edge, which is clearly more expensive.

Thus, a Nash equilibrium consists of all paths $p_{1}, p_{2}, \ldots, p_{\ell}$, and its cost equals $\ell$. In the optimal solution, all players are connected via path $p_{\ell}$. In order to connect some player belonging to path $p_{i}, i \neq \ell$, we use the edge of length $\frac{2}{q}$ connecting this player to path $p_{\ell}$. Note that the total number of players $M$ is bounded by $2 q k$, where $k$ is the number of vertices on path $p_{\ell}$. Clearly, $k \leq(\log q)^{\ell}$. Fixing $\ell=\frac{\log q}{\log \log q}$, we get $k \leq q$. The total cost of the optimal solution is less than $1+\frac{2 k}{q} \leq 3$ and the price of anarchy is therefore $\Omega(\ell)=\Omega\left(\frac{\log q}{\log \log q}\right)$. As $M \leq 2 q k \leq 2 q^{2}$, the price of anarchy is $\Omega(\ell)=\Omega\left(\frac{\log M}{\log \log M}\right)$.

We have thus proved the following theorem. 
Theorem 3: The cost of anarchy in the best-response dynamics of the integral multicast game is at least $\Omega(\log n / \log \log n)$.

\section{Intractability of Optimizing the Potential Function}

In this section we prove that finding a Nash equilibrium that minimizes the potential function is NP-hard. As a building block we use a variation of the Lund-Yannakakis proof [22] of hardness of approximation for the set cover problem.

The input to the set cover problem is a ground set of elements $U$ and a collection $\mathcal{S}$ of subsets of $U$. The goal is to choose a minimum cardinality collection of sets in $\mathcal{S}$ covering all elements. The reduction of [22] is performed from the 3SAT problem.

We use a straightforward and standard variation of the construction of [22] to obtain the following theorem (see, for instance Section 2 in [7], which contains the complete description of the construction and its analysis).

Theorem 4: Given a 3SAT formula $\varphi$, an instance of the set cover problem can be constructed in polynomial time, such that:

- All sets have equal size (denoted by $s$ ).

- If $\varphi$ is satisfiable (yes-instance), then there is a solution to the set cover instance that uses $X$ sets, and each element is covered by exactly one set in this solution.

- If $\varphi$ is not satisfiable (no-instance), then the size of any solution to the set cover instance is at least $\alpha X$, where $\alpha>1$ is some constant.

In the rest of this section we prove the following theorem:

Theorem 5: The problem of finding a Nash equilibrium of the integral multicast game that minimizes the potential function is NP-hard.

Proof: Given a 3SAT formula $\varphi$, we first construct a set cover instance as in Theorem 4, and then create an integral multicast game based on this instance, as follows. There is a vertex for each set and each element in the set cover problem, and additionally we have a special vertex $r$. The players are the vertices that represent the elements. Each vertex representing a set is connected to $r$ with a unit-length edge. Each vertex representing some element $i$ is connected to a vertex representing set $S$ if and only if $i \in \mathrm{S}$. The length of the edge is a large integer $q$, which will ensure that each user (element) chooses a path that contains only one such edge (i.e., connects via a set to which it belongs). Let $N$ denote the total number of users (elements) in the above example. It is enough to choose $q \geq N$.

Suppose $\varphi$ is a yes-instance. Then there is a solution $\mathcal{S}^{\prime}$ of size $X$ to the set cover instance. This solution naturally induces a Nash equilibrium in our game, where each element connects to the set that covers it in $\mathcal{S}^{\prime}$ and all the sets in $\mathcal{S}^{\prime}$ are connected to the root. Observe that there are exactly $s$ users on every edge that connects some set in $\mathcal{S}^{\prime}$ to the root. The value of the potential function in this solution is $q N+X \cdot H(s)$.

Assume now that $\varphi$ is a no-instance and suppose we are given some Nash equilibrium. This Nash equilibrium defines a solution to the set cover instance, since each element has to connect to one of the sets to which it belongs. However, the number of sets used in this solution is at least $\alpha X$, and some of the edges connecting these sets to the root are used by less than $s$ users. Thus, the value of the potential function in this solution is strictly greater than $q N+X \cdot H(s)$.

As determining whether a given 3SAT formula is satisfiable is NP-hard, it is NP-hard to find a Nash equilibrium minimizing the value of the potential function.

\section{The Fractional Multicast Game}

In this section we introduce a fractional model of the multicast game, where each user is allowed to split (fractionally) its connection to the source into several paths. The fractional model represents a splittable multicast model. While one unit of flow can be sent fractionally by the source to each user, the data (i.e., 
a flow fraction) is sent once on each edge of the multicast topology, independently from the number of its users. In the sequel we discuss how to efficiently implement this via network coding.

The cost of each flow fraction on an edge is evenly split between its users. Thus, the total cost of the flow on an edge is simply the cost of the flow fraction sent on that edge. We present our results for undirected graphs, yet they hold for directed graphs as well. In the fractional model, each user $i$ has to route one unit of flow from $t_{i}$ to the source $r$. User $i$ can split its unit of flow among any number of paths connecting $r$ to $t_{i}$. Denote the flow of user $i$ on edge $e$ by $f_{e, i}$ and the number of users on edge $e$ by $n_{e}$. Given a strategy profile $s$, assume without loss of generality that $f_{e, 1} \leq f_{e, 2} \leq \cdots \leq f_{e, n_{e}} \leq 1$. Define $f_{e, 0}=0$. Edge $e$ has capacity equal to $f_{e, n_{e}}$, and for convenience we think of the capacity of $e$ as defining an "address space" in the range $\left[0, f_{e, n_{e}}\right]$, where user $j$ uses $\left[0, f_{e, j}\right]$. The cost of each fraction of the capacity of $e$ is equally split between its users, as follows: $\left[f_{e, j-1}, f_{e, j}\right]$ is shared by $n_{e}-j+1$ users, where each user pays $c_{e} \frac{f_{e, j}-f_{e, j-1}}{n_{e}-j+1}$. Therefore, the total cost $c_{e}^{i}$ paid by user $i$ for the use of edge $e$ is:

$$
c_{e}^{i}=c_{e} \cdot \sum_{k=1}^{i} \frac{f_{e, k}-f_{e, k-1}}{n_{e}-k+1} .
$$

As the total flow fraction sent on edge $e$ is $f_{e, n_{e}}$, the total cost of the edge is simply $c_{e} \cdot f_{e, n_{e}}$.

We denote by $P^{i}$ the set of paths used by user $i$. The cost of a path $p \in P^{i}$ is the sum of its edge costs, that is $\sum_{e \in p} c_{e}^{i}$. The total cost $c^{i}$ of a user $i$ is the sum of its path costs, that is $\sum_{p \in P^{i}} \sum_{e \in p} c_{e}^{i}$. Each user $i$ aims to establish its flow from the source $r$ to $t_{i}$ so as to minimize its cost. Thus, a flow $f$ is at Nash equilibrium if no user has any incentive of changing its flow to the root. An instance of the fractional model, consisting of a graph $G$, a source $r$, a set of receivers $N$, and a cost vector $c$ is denoted by $\operatorname{frac}(G, r, N, c)$. We introduce a potential function $\Phi$ for the fractional multicast game which is based on Rosenthal's potential function [28], as follows:

$$
\Phi=\sum_{e \in s}\left(\sum_{j=1}^{n_{e}(s)} \sum_{i=1}^{n_{e}+1-j} c_{e} \frac{f_{e, j}-f_{e, j-1}}{i}\right) .
$$

The proof of the next theorem follows from the proof of Rosenthal's potential function [28].

Theorem 6: The potential fuction $\Phi$ is an exact potential for the fractional multicast game. That is, for every $k \in N$, and every pair of strategy profiles $\left(s^{-k}, s_{1}^{k}\right)$ and $\left(s^{-k}, s_{2}^{k}\right)$, if $c^{k}\left(s^{-k}, s_{1}^{k}\right)<c^{k}\left(s^{-k}, s_{2}^{k}\right)$, where $c^{k}$ denotes the total cost of user $k$, then

$$
c^{k}\left(s^{-k}, s_{2}^{k}\right)-c^{k}\left(s^{-k}, s_{1}^{k}\right)=\Phi\left(s^{-k}, s_{2}^{k}\right)-\Phi\left(s^{-k}, s_{1}^{k}\right) .
$$

The proof of Theorem 6 appears in Appendix A1. As a fractional flow configuration defining a local minimum of the potential function constitutes a Nash equilibrium, we get:

Theorem 7: A Nash equilibrium exists for every instance $\operatorname{frac}(G, r, N, c)$.

We now explain how a fractional solution can be implemented via network coding. A Nash equilibrium configuration determines a directed flow network (note that if an edge is used in both directions, then it should be replaced by two directed edges). We define the capacity of a cut separating $r$ from a terminal $t_{i} \in N$ as the sum of the capacities of the forward edges in the cut, where the capacity of edge $e$ is $f_{e, n_{e}}$. By allowing each user $i$ to split its connection to $r$ as described above, it follows that the capacity of any cut separating $r$ from $t_{i} \in N$ is at least 1. Koetter and Medard [18] (see also [3], [21]) showed that the transmission rate of a fractional multicast transmission is bounded by the capacity of the minimum cut (taken over all $i$ ) separating $r$ from $t_{i} \in N$, and this bound is tight. This means that in our case, where the capacity of the minimum cut is at least 1 , the transmission rate is the same as in a tree. Therefore, a Nash equilibrium of the fractional game is a stable operating point, where the users share the cost of a solution achieving the minimum cut property and no player can unilaterally reduce its cost by changing its strategy. 


\section{A. Computing a Minimum Potential Nash Equilibrium}

We proceed to describe how a Nash equilibrium of the fractional game can be computed in polynomial time using linear programming. Moreover, the computed Nash equilibrium minimizes the potential function $\Phi$. Compare that with the hardness of finding an integral solution minimizing the potential function. In addition, as shown later, the minimum potential Nash equilibrium is within a factor of $O(\log n)$ away from the cost of an optimal fractional Steiner tree.

Given an instance $\operatorname{frac}(G, r, N, c)$, we create a new graph $G^{\prime}=\left(V, E^{\prime}\right)$ by replacing each edge $e$ by $n$ copies $e_{1}, e_{2}, \ldots, e_{n}$. The cost of a unit flow on edge $e_{j}$ is $c_{e} / j$. For a path $p$ from $t_{i}$ to $r$ in $G^{\prime}$, we denote by $f_{p}^{i}$ the amount of flow of commodity $i$ sent on it. Note that different paths can use an edge in opposite directions.

We formulate a linear program with an objective function that is equal to the potential of the fractional multicast game. The variables of the linear program are the flows of the users sent on the set of paths in $G^{\prime}$ from the terminals $t_{1}, \ldots, t_{n}$ to the root $r$, and the capacities of the edges in $E^{\prime}$. Denote a path from $t_{i}$ to $r$ by $t_{i} \rightsquigarrow r$. The capacity of edge $e_{j}$ is denoted by $x_{e_{j}}$, where $0 \leq x_{e_{j}} \leq 1$. The linear program is as follows.

$$
\begin{aligned}
& \text { minimize } \sum_{e \in E} \sum_{j=1}^{n}\left(\sum_{i=1}^{j} \frac{c_{e} \cdot x_{e_{j}}}{i}\right) \quad \text { s.t. } \\
& \text { For each commodity } i: \sum_{p: t_{i} \rightsquigarrow r} f_{p}^{i} \geq 1 \\
& \text { For each edge } e \text {, copy } j \text {, commodity } i: \sum_{p: t_{i} \rightsquigarrow r \mid e_{j} \in p} f_{p}^{i} \leq x_{e_{j}} \\
& \text { For each edge } e, \text { copy } j: \sum_{i=1}^{n} \sum_{p: t_{i} \rightsquigarrow r \mid e_{j} \in p} f_{p}^{i}=j \cdot x_{e_{j}} \\
& 0 \leq x_{e_{j}} \leq 1, \quad f_{p}^{i} \geq 0
\end{aligned}
$$

The total flow of user $i$, summed up over all paths from $t_{i}$ to $r$, is at least 1 (Constraint (1)). Constraint (2), the non-aggregating flow constraint, restricts the flow of each user $i$ on edge $e_{j}$ to be at most its capacity $x_{e_{j}}$. The total flow, taken over all commodities on edge $e_{j}$, is constrained to be precisely $j \cdot x_{e_{j}}$, as restricted by Constraint (3), the aggregating flow constraint. This constraint is satisfied in the integral case: if $j$ commodities are sent on edge $e$, then edge $e_{j}$ is "bought", and the number of users on this edge is $j$. The sum of the costs of the commodities on $e_{j}$ is then exactly $c_{e}$.

Note that the above linear program uses an exponential number of variables. However, it can be solved in polynomial time via the dual program using the Ellipsoid algorithm. Alternatively, it can be formulated with a polynomial number of variables by using the flows of the users on the different edges in $G^{\prime}$ as variables.

1) Characterizing an Optimal Solution: We say that a flow $f$ on instance $G^{\prime}$ is canonical if it has, for every edge $e \in E$, the following structure. Denote by $f_{e, j}$ the sum of the flows of user $j$ on all copies of $e\left(f_{e, j}=\sum_{k=1}^{n} \sum_{p: t_{j} \rightsquigarrow r \mid e_{k} \in p} f_{p}^{j}\right)$. Suppose that without loss of generality $f_{e, 1} \leq f_{e, 2} \leq \cdots \leq f_{e, n_{e}} \leq 1$, where $n_{e}$ denotes the number of users with positive flow. Then, the flows routed on $e_{n_{e}}, \ldots, e_{2}, e_{1}$ are $f_{e, 1},\left(f_{e, 2}-f_{e, 1}\right), \ldots,\left(f_{e, j}-f_{e, j-1}\right), \ldots,\left(f_{e, n_{e}}-f_{e, n_{e}-1}\right)$, respectively, and the non-aggregating flow on copies $e_{i}$ for $i \geq n_{e}+1$ is zero. Notice that there is a one-to-one correspondence between canonical flows in the instance $G^{\prime}$ and fractional multicast flows in $G$. We now turn to prove that there exists a canonical flow minimizing the potential function.

Let $f$ be the output flow of the linear program. We first consider the flow $f_{e_{k}}$ on each copy $e_{k}$ of edge $e$, and rearrange it to be a canonical flow. Then, we merge these resulting canonical flows into a single 
canonical flow on $e$. These two steps are performed for each edge $e \in E$. We show that the resulting potential of the new (canonical) flow is not larger than the potential of the original flow $f$.

Lemma 4.1: Consider edge $e_{k} \in E^{\prime}, 1 \leq k \leq n$. There exists a canonical flow on $e_{k}$ with potential value not greater than that of the original flow on $e_{k}$.

Proof: Without loss of generality, suppose that $f_{e_{k}, 1}<f_{e_{k}, 2}<\cdots<f_{e_{k}, \ell}$ are the different amounts of flow routed on $e_{k}$ by the users, where $f_{e_{k}, \ell}=x_{e_{k}}$. For ease of notation, we denote this ordering as $f_{1}<f_{2}<\cdots<f_{\ell}$, where $f_{\ell}=x_{e_{k}}$. Assume that the number of users routing a flow value $\leq f_{i}$ is $k_{i}$, and thus $k_{1}>k_{2}>\cdots>k_{\ell}$. We rearrange the flow $f_{e_{k}}$ to be a canonical flow by sending each amount of flow to its proper edge copy, i.e. by "buying" capacity $f_{1}$ on edge $e_{k_{1}}$, capacity $\left(f_{2}-f_{1}\right)$ on edge $e_{k_{2}}$, etc.

The potential of the resulting canonical flow derived from $f_{e_{k}}$ is thus

$$
c_{e} \sum_{i=1}^{\ell} \sum_{j=1}^{k_{i}} \frac{f_{i}-f_{i-1}}{j}=c_{e} \sum_{i=1}^{\ell}\left(f_{i}-f_{i-1}\right) H\left(k_{i}\right),
$$

where $f_{0}=0$. On the other hand, the potential of the original flow on $e_{k}$ is

$$
c_{e} \sum_{i=1}^{k} \frac{x_{e_{k}}}{i}=c_{e} \cdot H(k) x_{e_{k}}=c_{e} \cdot H(k) f_{\ell} .
$$

The total flow on $e_{k}$ is constrained to be $k \cdot x_{e_{k}}=k \cdot f_{\ell}$ (Constraint (3)), which is equal to the total canonical flow derived from $f_{e_{k}}$, and thus

$$
k \cdot f_{\ell}=\sum_{i=1}^{\ell} k_{i}\left(f_{i}-f_{i-1}\right) .
$$

Since $0 \leq \frac{\left(f_{i}-f_{i-1}\right)}{f_{\ell}} \leq 1$ and $\sum_{i=1}^{\ell} \frac{\left(f_{i}-f_{i-1}\right)}{f_{\ell}}=1$, by Jensen's inequality,

$$
\sum_{i=1}^{\ell} H\left(k_{i}\right) \frac{f_{i}-f_{i-1}}{f_{\ell}} \leq H\left(\sum_{i=1}^{\ell} k_{i} \cdot \frac{f_{i}-f_{i-1}}{f_{\ell}}\right)=H(k),
$$

and thus $\sum_{i=1}^{\ell} H\left(k_{i}\right)\left(f_{i}-f_{i-1}\right) \leq H(k) f_{\ell}$.

Lemma 4.2: Consider edge $e \in E$ and two canonical flows $f_{e}$ and $f_{e}^{\prime}$. Then $f_{e}$ and $f_{e}^{\prime}$ can be added up yielding a canonical flow with potential value not greater than the sum of the potentials of $f_{e}$ and $f_{e}^{\prime}$.

Proof: Consider two canonical flows $f$ and $f^{\prime}$, and assume that their respective flows on $e_{k}$ are $x_{k}$ and $x_{k}^{\prime}$. That is, $x_{k}$ (resp., $x_{k}^{\prime}$ ) is the amount of flow routed by each player using $e_{k}$ according to $f$ (resp., $f^{\prime}$ ). We denote by $G_{k}$ and $G_{k}^{\prime}$ the sets of players that use $e_{k}$ according to $f$ and $f^{\prime}$ respectively, where $\left|G_{k}\right|=\left|G_{k}^{\prime}\right|=k$. Assume, without loss of generality that $x_{k}^{\prime} \geq x_{k}$. By merging these two flows into a single canonical flow, we "buy" capacity $x_{k}$ on copy $\left|G_{k} \cup G_{k}^{\prime}\right|$ of edge $e$, capacity $\left(x_{k}^{\prime}-x_{k}\right)$ on $e_{k}$, and capacity $x_{k}$ on copy $\left|G_{k} \cap G_{k}^{\prime}\right|$ of edge $e$. We thus get a new canonical flow with potential

$$
H\left(\left|G_{k} \cup G_{k}^{\prime}\right|\right) x_{k}+H(k)\left(x_{k}^{\prime}-x_{k}\right)+H\left(\left|G_{k} \cap G_{k}^{\prime}\right|\right) x_{k} .
$$

On the other hand, the sum of potentials of the original flows on $e_{k}$ is $H(k) x_{k}+H(k) x_{k}^{\prime}$. As

$$
2 H(k) \geq H\left(\left|G_{k} \cup G_{k}^{\prime}\right|\right)+H\left(\left|G_{k} \cap G_{k}^{\prime}\right|\right),
$$

we get that the potential of the new canonical flow is not larger than the sum of potentials of the original flows.

In case $G_{k} \neq G_{k}^{\prime}$, the potential of the new canonical flow is strictly less than the sum of the potentials of the original canonical flows. In this case, capacity has to be bought on other copies of $e$ except for $e_{k}$, and thus other merging steps should be performed for each such copy. As each such step strictly decreases 
the potential, the merging process is finite.

Theorem 8: There exists an optimal solution to the linear program which is a canonical flow.

Proof: Let $f$ be the output flow of the linear program. As $f$ is a flow of minimum potential, it is either canonical, or can be easily rearranged as such by performing the two steps described by Lemmas 4.1 and 4.2 on all copies of each edge $e$.

The linear program presented for computing the minimum potential Nash equilibrium of the fractional model can be used for more general settings, not necessarily egalitarian, where the cost sharing mechanisms are cross-monotonic, i.e. the cost functions are non-increasing in the number of users. Furthermore, it can also be used for settings where the users are not restricted to have a common source. Recall that finding an integral solution with minimum potential is NP-hard.

There are instances for which there is a gap between the minimum potential fractional Nash equilibrium and the minimum potential integral Nash equilibrium. Consider Figure 3, which depicts an instance $\left(G, r,\left\{t_{1}, t_{2}, t_{3}\right\}, c\right)$, for which the minimum potential fractional Nash equilibrium is smaller than the minimum potential integral Nash equilibrium. The edge costs are as follows: the cost of each edge $\left(r, v_{i}\right)$ $(i=1,2,3)$ is $x$, and the cost of each edge from $v_{i}$ to the terminals connected to it is $q \gg x$. The fractional Nash equilibrium that minimizes the potential is as follows: each terminal $t_{i}$ sends $1 / 2$ unit of flow through each of the two vertices $v_{j}(j=1,2,3)$ connecting it to $r$. Therefore, the fractional potential is $\Phi_{\text {frac }}=3 x / 2(1+1 / 2)+6 q / 2=9 x / 4+3 q$. On the other hand, the integral Nash equilibrium that minimizes the potential is as follows: two out of the three terminals send their flow through the same vertex $v_{i}$ to $r$, and the third terminal sends its flow through one out of the other two vertices $v_{j}$ connecting it to $r$. Therefore, the integral potential is $\Phi_{\text {int }}=x(1+1 / 2)+x+3 q=10 x / 4+3 q$.

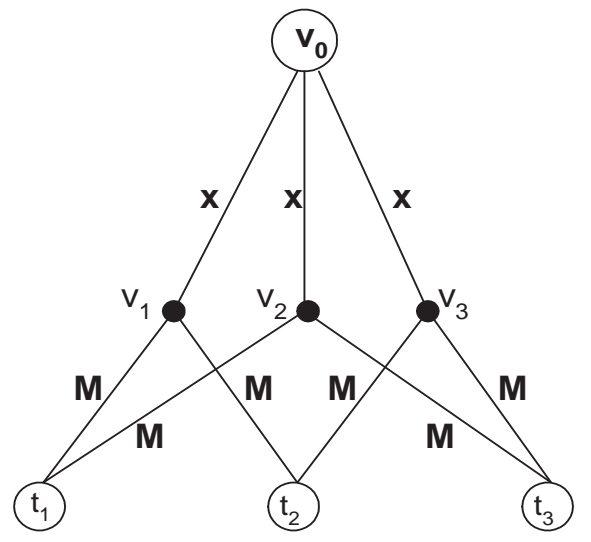

Fig. 3. Instance in which the minimum potential fractional Nash equilibrium is smaller than the minimum potential integral Nash equilibrium.

We define the price of anarchy of the fractional game as the ratio between the cost of a Nash equilibrium solution and the cost of an optimal fractional solution to the Steiner tree problem.

We show that the price of anarchy of a minimum potential fractional Nash equilibrium solution is $O(\log n)$. This follows since this solution has potential that is not higher than the potential of an optimal fractional Steiner tree, and the potential of a solution is within at most a factor of $O(\log n)$ away from its cost. Also, note that the potential of a solution is an upper bound on its cost.

More formally, for any fractional solution $T$, let $\Phi(T)$ denote the value of its potential function and let $C(T)$ denote the total cost of edges participating in $T$. We denote by $T_{\text {Nash }}$ the fractional Nash equilibrium tree with minimum potential, and by $T_{\text {Steiner }}$ the fractional optimal Steiner tree. We get that

$$
C\left(T_{\text {Nash }}\right) \leq \Phi\left(T_{\text {Nash }}\right) \leq \Phi\left(T_{\text {Steiner }}\right) \leq \log n \cdot C\left(T_{\text {Steiner }}\right)
$$

The first and third inequalities follow from the properties of the potential function, where each fraction of flow $f_{e, j}$ on an edge $e$ is multiplied by $1 \leq H(k) \leq \log n$, and $k, 1 \leq k \leq n$, is the number of users 
generating this flow fraction. The second inequality follows from the fact that the potential value of $T_{\text {Nash }}$ is minimal.

We observe that the $\Omega\left(\frac{\log n}{\log \log n}\right)$ lower bound on the price of anarchy for the integral model holds also for the fractional model. That is, the ratio of the cost of some Nash equilibrium (not necessarily the one that minimizes the potential function) and the Steiner tree cost can be as large as $\Omega\left(\frac{\log n}{\log \log n}\right)$.

\section{B. The Weighted Fractional Multicast Game}

We consider a weighted extension of our multicast game, where each user $i$ is associated with a positive weight $w_{i}$. The payment of each player is proportional to its weight. Let $W_{j}=\sum_{i=1}^{j} w_{i}$. Given a flow vector $f$, let $f_{e, i}$ denote the flow of user $i$ on $e$ and let $n_{e}$ be the number of users with non-zero flow on $e$. Assume that the users are numbered such that $0=f_{e, 0}<f_{e, 1} \leq f_{e, 2} \leq \ldots \leq f_{e, n_{e}}$. Consider some $j \leq n_{e}$. User $j$ shares the capacity $f_{e, 1}$ with users 1 to $n_{e}$, shares $f_{e, 2}-f_{e, 1}$ with users 2 to $n_{e}$ and so on. The payment for any share is in proportion to weights. Hence, for the capacity $f_{e, 1}$, user $j \leq n_{e}$ pays $c_{e} \cdot f_{e, 1} \cdot w_{j} / W_{n_{e}}$. Thus the overall cost paid by $j \leq n_{e}$ on edge $e$ is

$$
c_{e} \cdot w_{j} \cdot \sum_{i=1}^{j} \frac{f_{e, i}-f_{e, i-1}}{W_{n_{e}}-W_{i-1}} .
$$

The overall payment of a user is the sum of its payments for the flow fractions it uses on all edges in all its paths. Each user $j$ aims to establish its flow from the source $r$ to $t_{j}$ so as to minimize its cost. Thus, a flow $f$ is at Nash equilibrium if no user has an incentive to change its flow.

An instance of the weighted fractional model, consisting of a graph $G$, a source $r$, a set of receivers $N$ with weight vector $w$, and a cost vector $c$ is denoted by $\operatorname{frac}(G, r, N, c, w)$. The proof of the following theorem uses Kakutani's fixed point theorem [17], and appears in Section A2 of the Appendix.

Theorem 9: A Nash equilibrium (in pure strategies) exists for every instance $\operatorname{frac}(G, r, N, c, w)$.

This theorem should be contrasted with the result of Chen and Roughgarden [6] who showed that a Nash equilibrium does not necessarily exist in the integral weighted multicast game.

\section{REFERENCES}

[1] M. Adler, D. Rubenstein, "Pricing multicasting in more practical network models", Proc. of ACM-SIAM SODA, 981-990, 2002.

[2] A. Agarwal and M. Charikar, "On the advantage of network coding for improving network throughput", Proc. of IEEE Information Theory Workshop, 2004.

[3] R. Ahlswede, N. Cai, S.-Y. R. Li and R. W. Yeung, "Network information flow", IEEE Transactions on Information Theory, Vol. 46, 1204-1216, 2000.

[4] E. Anshelevich, A. Dasgupta, J. Kleinberg, É. Tardos, T. Wexler and T. Roughgarden, "The price of stability for network design with fair cost allocation", Proc. of IEEE FOCS, 295-304, 2004.

[5] A. Archer, J. Feigenbaum, A. Krishnamurthy, R. Sami and S. Shenker, "Approximation and collusion in multicast cost sharing", Proc. of ACM EC, 253-255, 2001.

[6] H.-L. Chen and T. Roughgarden. Network design with weighted players. Proceedings of the 18th Annual ACM Symposium on Parallelism in Algorithms and Architectures, 2006, pp. 29-38.

[7] J. Chuzhoy, A. Gupta, S. Naor and A. Sinha. On the Approximability of Some Network Design Problems. ACM Transactions on Algorithms (TALG), to appear. Preliminary version appeared in Proc. 16th Annual ACM-SIAM Symposium on Discrete Algorithms (SODA), pp. 943-951, 2005.

[8] A. Fabrikant, C. Papadimitriou and K. Talwar, "The complexity of pure nash equilibria", Proc. of ACM STOC, 604-612, 2004.

[9] J. Feigenbaum, C. Papadimitriou and S. Shenker, "Sharing the cost of multicast transmission", Proc. of ACM STOC, 218-227, 2000.

[10] A. Fiat, H. Kaplan, M. Levy, S. Olonetsky and R. Shabo, "On the price of stability for designing undirected networks with fair cost allocations", to appear in ICALP '06.

[11] C.H. Helvig, G. Robins, and A. Zelikovsky, "Improved approximation scheme for the group Steiner problem", Networks, Vol. 37(1), 8-20, 2001.

[12] S. Herzog, S. Shenker and D. Estrin, "Sharing the cost of multicast trees: An axiomatic analysis", IEEE/ACM Transactions on Networking, Vol. 5, 847-860, 1997.

[13] R. Holzman and N. Law-Yone, "Strong equilibrium in congestion games", Games and Economic Behavior, Vol. 21, 85-101, 1997.

[14] M. Imaze and B. Waxman, "Dynamic steiner tree problem", SIAM J. on Discrete Mathematics, Vol. 4(3), 369-384, 1991.

[15] K. Jain and V. Vazirani, "Applications of approximation algorithms to cooperative games", Proc. of the ACM STOC, 364-372, 2001.

[16] R. Johari and J.N. Tsitsiklis, "Efficiency loss in a network resource allocation game", to appear in Math. of Oper. Res. 
[17] S. Kakutani, “A generalization of Brouwer's Fixed Point Theorem”, Duke Mathematical Journal, Vol. 8, 457-459, 1941.

[18] R. Koetter and M. Medard, "An algebraic approach to network coding", IEE/ACM Transactions on Networking, Vol. 11(5), 782-795, 2003.

[19] Y.A. Korilis, A.A. Lazar and A. Orda, "Achieving network optima using Stackelberg routing strategies", IEEE/ACM Transactions on Networking, Vol. 5(1), 161-173, 1997.

[20] E. Koutsoupias and C. Papadimitriou, "Worst-case equilibria", Proc. of STACS, LNCS, 404-413, 1999.

[21] S.-Y. R. Li, R. W. Yeung, and N. Cai, "Linear network coding”, IEEE Transactions on Information Theory, Vol. 49, 371-381, 2003.

[22] C. Lund and M. Yannakakis, "On the hardness of approximating minimization problems", JACM, Vol. 41(5), 960-981, 1994.

[23] I. Milchtaich, "Congestion games with player specific payoff functions", Games and Economic Behavior, Vol. 13, 111-124, 1996.

[24] V. Mirrokni and A. Vetta, "Convergence issues in competitive games", Proc. of APPROX, LNCS, 183-194, 2004.

[25] D. Monderer and L.S. Shapley, "Potential games", Games and Economic Behavior, Vol. 14, 124-143, 1996.

[26] A. Orda, R. Rom and N. Shimkin, "Competitive routing in multi-user communication networks", IEEE/ACM Transaction on Networking, Vol. 1, 614-627, 1993.

[27] C. Papadimitriou, "Algorithms, games, and the Internet", Proc. of ACM STOC, 749-753, 2001.

[28] R.W. Rosenthal, "A class of games possessing pure strategy Nash equilibria", International Journal of Game Theory, Vol. 2, 65-67, 1973.

[29] T. Roughgarden and É. Tardos, "How bad is selfish routing?", JACM, Vol. 49(2), 236-259, 2002.

[30] T. Roughgarden, "The price of anarchy is independent of the network topology", Proc. of ACM STOC, 428-437, 2002.

[31] L.S. Shapley, 'A value for n-person games", Contribution to the Theory of Games, 31-40, Princeton University Press, Princeton, 1953.

[32] A.S. Schulz and N.E. Stier Moses, "On the performance of user equilibria in traffic networks", Proc. of ACM-SIAM SODA, 86-87, 2003.

[33] M. Voorneveld, P. Borm, F. van Megen, S. Tijs and G. Faccini, "Congestion games and potentials reconsidered", International Game Theory Review, Vol. 1, 283-299, 1999.

[34] A. Zelikovsky, "A series of approximation algorithms for the acyclic directed steiner tree problem", Algorithmica, Vol. 18, 99-110, 1997.

\section{APPENDIX}

\section{A1. Proof of THEOREM 6}

Theorem 6. The potential fuction $\Phi$ is an exact potential for the fractional multicast game. That is, for every $k \in N$, and every pair of strategy profiles $\left(s^{-k}, s_{1}^{k}\right)$ and $\left(s^{-k}, s_{2}^{k}\right)$, if $c^{k}\left(s^{-k}, s_{1}^{k}\right)<c^{k}\left(s^{-k}, s_{2}^{k}\right)$, where $c^{k}$ denotes the total cost of user $k$, then

$$
c^{k}\left(s^{-k}, s_{2}^{k}\right)-c^{k}\left(s^{-k}, s_{1}^{k}\right)=\Phi\left(s^{-k}, s_{2}^{k}\right)-\Phi\left(s^{-k}, s_{1}^{k}\right) .
$$

Proof:

Consider a player $k \in N$ and strategies $s^{-k} \in S^{-k}$, and $s^{k},\left(s^{k}\right)^{*} \in S^{k}$ such that $c^{k}\left(s^{-k},\left(s^{k}\right)^{*}\right)<$ $c^{k}\left(s^{-k}, s^{k}\right)$. We denote by $s$ and $s^{*}$ the strategy profiles $\left(s^{-k}, s^{k}\right)$ and $\left(s^{-k},\left(s^{k}\right)^{*}\right)$ respectively, and by $n_{e}$ and $n_{e}^{*}$ the number of players using edge $e$ according to the strategy profiles $s$ and $s^{*}$ respectively. As before, we generate an ordering $f_{e, 1} \leq f_{e, 2} \leq \cdots \leq f_{e, n_{e}} \leq 1$ on the flow $f_{e}$ routed by the $n_{e}$ users on $e$ according to $s$ and an ordering $f_{e, 1}^{*} \leq f_{e, 2}^{*} \leq f_{e, n_{e}^{*}}^{*} \leq 1$ on the flow $f_{e}^{*}$ routed by the $n_{e}^{*}$ users on $e$ according to $s^{*}$. We assume that $f_{e, 0}=f_{e, 0}^{*}=0$. We denote by $f_{e}^{k}$ and $\left(f_{e}^{k}\right)^{*}$ the flow of user $k$ on edge $e$ according to strategies $s^{k}$ and $\left(s^{k}\right)^{*}$ respectively. Assuming $f_{e}^{k}>0, i_{e}^{k}$ denotes the index of user $k$ 's flow on edge $e$ according to this ordering, otherwise $i_{e}^{k}=0$. Similarly, $\left(i_{e}^{k}\right)^{*}$ is the index of user $k$ 's flow on edge $e$ assuming $\left(f_{e}^{k}\right)^{*}>0$, and equals 0 otherwise. We now compare the total cost $c^{k}$ of user $k$ according to $s$ and the corresponding cost $\left(c^{k}\right)^{*}$ according to $s^{*}$ :

$$
\begin{aligned}
\left(c^{k}\right)^{*} & =\sum_{e \in\left(s^{k}\right)^{*}}\left(\sum_{j=1}^{\left(i_{e}^{k}\right)^{*}} c_{e} \cdot \frac{f_{e, j}^{*}-f_{e, j-1}^{*}}{n_{e}^{*}+1-j}\right) \\
& <\sum_{e \in s^{k}}\left(\sum_{j=1}^{i_{e}^{k}} c_{e} \cdot \frac{f_{e, j}-f_{e, j-1}}{n_{e}+1-j}\right)=c^{k} .
\end{aligned}
$$




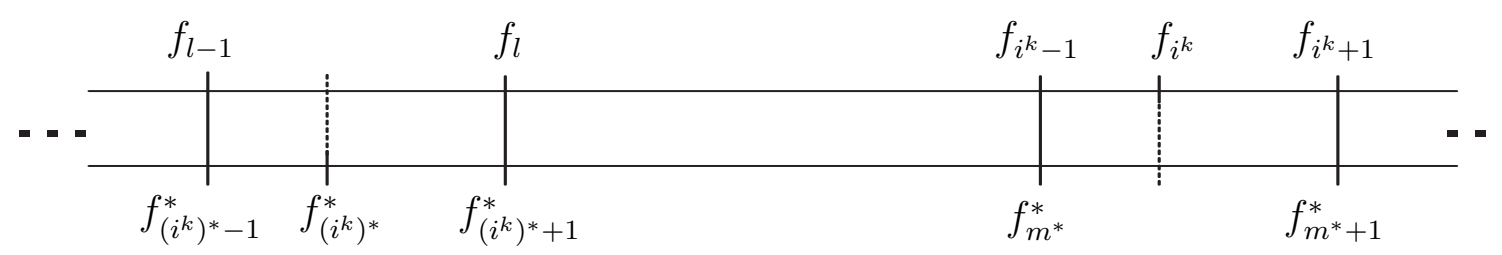

Fig. 4. Flow on edge $e$ in case $f_{e}^{k}>\left(f_{e}^{k}\right)^{*}$ (for simplicity, subscripts of $e$ are omitted).

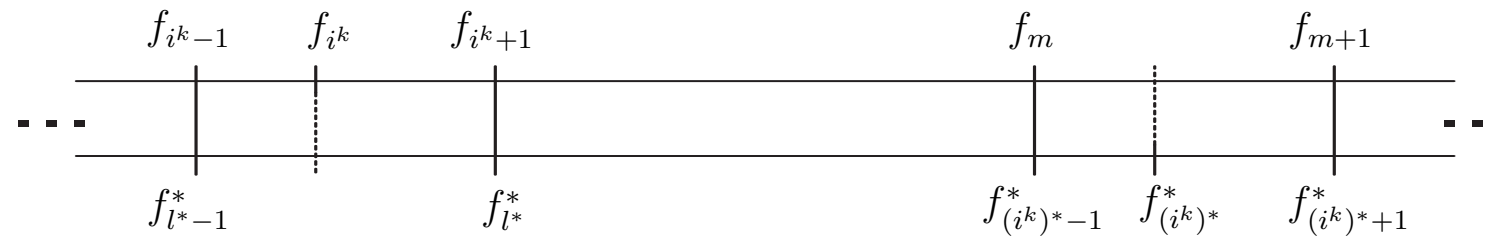

Fig. 5. Flow on edge $e$ in case $f_{e}^{k}<\left(f_{e}^{k}\right)^{*}$ (for simplicity, subscripts of $e$ are omitted).

We can split each sum on both sides of the above inequality to two sums, one sum describing the flows that are equal according to $\left(s^{-k}, s^{k}\right)$ and $\left(s^{-k},\left(s^{k}\right)^{*}\right)$, and the other sum describing the flows that differ according to these two strategy profiles. We thus remain with the differing flows:

$$
\begin{array}{r}
\sum_{\left\{e \mid f_{e}^{k}<\left(f_{e}^{k}\right)^{*}\right\}} c_{e} \cdot\left(\frac{f_{e, l_{e}^{*}}^{*}-f_{e, i_{e}^{k}}}{n_{e}^{*}+1-l_{e}^{*}}+\sum_{j=l_{e}^{*}+1}^{\left(i_{e}^{k}\right)^{*}} \frac{f_{e, j}^{*}-f_{e, j-1}^{*}}{n_{e}^{*}+1-j}\right) \\
<\sum_{\left\{e \mid f_{e}^{k}>\left(f_{e}^{k}\right)^{*}\right\}} c_{e} \cdot\left(\frac{f_{e, l_{e}}-f_{e,\left(i_{e}^{k}\right)^{*}}^{*}}{n_{e}+1-l_{e}}+\sum_{j=l_{e}+1}^{i_{e}^{k}} \frac{f_{e, j}-f_{e, j-1}}{n_{e}+1-j}\right)
\end{array}
$$

where, for $f_{e}^{k}>\left(f_{e}^{k}\right)^{*}, l_{e}$ is the index of the flow for which $f_{e, l_{e}}=f_{e,\left(i_{e}^{k}\right)^{*}+1}^{*}$ according to the ordering of $f_{e}$, while for $f_{e}^{k}<\left(f_{e}^{k}\right)^{*}, l_{e}^{*}$ is the index of the flow for which $f_{e, l_{e}^{*}}^{*}=f_{e, i_{e}^{k}+1}$ according to the ordering of $f_{e}^{*}$ (see Figures 4 and 5).

Note that, for an edge $e$ where $f_{e}^{k}=f_{e, i_{e}^{k}}<f_{e,\left(i_{e}^{k}\right)^{*}}^{*}=\left(f_{e}^{k}\right)^{*}$, it holds that the number of users using each segment in the range $\left[f_{e, i_{e}^{k}}, f_{e,\left(i_{e}^{*}\right)^{*}}\right]$ according to the ordering of $f_{e}^{*}$ is larger by one than the number of users using each segment in this range according to the ordering of $f_{e}$, as user $k$ is the only user changing its flow. We thus get:

$$
\begin{aligned}
& \sum_{\left\{e \mid f_{e}^{k}<\left(f_{e}^{k}\right)^{*}\right\}} c_{e} \cdot\left(\sum_{j=i_{e}^{k}+1}^{m_{e}} \frac{f_{e, j}-f_{e, j-1}}{n_{e}+2-j}+\frac{f_{e,\left(i_{e}^{k}\right)^{*}}^{*}-f_{e, m_{e}}}{n_{e}+2-\left(m_{e}+1\right)}\right) \\
< & \sum_{\left\{e \mid f_{e}^{k}>\left(f_{e}^{k}\right)^{*}\right\}} c_{e} \cdot\left(\frac{f_{e, l_{e}}-f_{e,\left(i_{e}^{k}\right)^{*}}^{*}}{n_{e}+1-l_{e}}+\sum_{j=l_{e}+1}^{i_{e}^{k}} \frac{f_{e, j}-f_{e, j-1}}{n_{e}+1-j}\right),
\end{aligned}
$$

where for $f_{e}^{k}<\left(f_{e}^{k}\right)^{*}, m_{e}$ is the index of the flow for which $f_{e, m_{e}}=f_{e,\left(i_{e}^{k}\right)^{*}-1}^{*}$ (see Figure 5). We now compare the potential $\Phi(s)$ with the potential $\Phi\left(s^{*}\right)$. 


$$
\begin{aligned}
\Phi\left(s^{*}\right)= & \sum_{e \in s^{*}}\left(\sum_{j=1}^{n_{e}^{*}} \sum_{i=1}^{n_{e}^{*}+1-j} c_{e} \frac{f_{e, j}^{*}-f_{e, j-1}^{*}}{i}\right) \\
= & \sum_{e \in s}\left(\sum_{j=1}^{n_{e}} \sum_{i=1}^{n_{e}+1-j} c_{e} \frac{f_{e, i}-f_{e, i-1}}{i}\right)+ \\
& \sum_{\left\{e \mid f_{e}^{k}<\left(f_{e}^{k}\right)^{*}\right\}} c_{e} \cdot\left(\sum_{j=i_{e}^{k}+1}^{m_{e}} \frac{f_{e, j}-f_{e, j-1}}{n_{e}+2-j}+\frac{f_{e,\left(i_{e}^{k}\right)^{*}}^{*}-f_{e, m_{e}}}{n_{e}+2-\left(m_{e}+1\right)}\right)- \\
& \sum_{\left\{e \mid f_{e}^{k}>\left(f_{e}^{k}\right)^{*}\right\}} c_{e} \cdot\left(\frac{f_{e, l_{e}}-f_{e,\left(i_{e}^{k}\right)^{*}}}{n_{e}+1-l_{e}}+\sum_{j=l_{e}+1}^{i_{e}^{k}} \frac{f_{e, j}-f_{e, j-1}}{n_{e}+1-j}\right) \\
< & \sum_{e \in s}\left(\sum_{j=1}^{n_{e}} \sum_{i=1}^{n_{e}+1-j} c_{e} \frac{f_{e, j}-f_{e, j-1}}{i}\right)=\Phi(s)
\end{aligned}
$$

Therefore, it follows that $c^{k}-\left(c^{k}\right)^{*}=\Phi(s)-\Phi\left(s^{*}\right)$.

\section{A2. Proof of TheOREM 9}

Theorem 9. A Nash equilibrium exists for every instance $\operatorname{frac}(G, r, N, c, w)$.

For each player $i$ we define a best reply correspondence $\Psi^{i}$ from $S$ to $S^{i}$ as follows. For any $s \in S$, let

$$
\Psi^{i}(s)=\left\{s^{i} \in S^{i} \mid c^{i}\left(s^{-i}, s^{i}\right) \geq c^{i}\left(s^{-i}, \widetilde{s^{i}}\right) \text { for every } \widetilde{s^{i}} \in S^{i}\right\}
$$

Each set $S^{j}$ consists of player $j$ 's possible flow patterns from $r$ to $d_{j}$. Thus, it is nonempty, compact (finite and closed), and convex. It follows that the set $S$ is a compact and convex subset of Euclidian space, since each $S^{j}$ is such.

By definition, $\Psi^{i}(s)$ is the set of strategies that minimizes $i$ 's cost given the strategies of the other players prescribed by $s$. According to the definition of our model, this set is nonempty as it represents user $i$ 's minimum cost sets of paths, given the flow pattern of the other users.

We prove the existence of a Nash equilibrium for every instance of a fractional multicast game. In order to establish the proof, we first consider several properties of $\Psi^{i}$. We show that the correspondence $\Psi^{i}$ is convex-valued and upper hemicontinuous.

Lemma 1.1: The correspondence $\Psi^{i}$ is convex-valued.

Proof: We prove that, given two strategies $s_{1}^{i}, s_{2}^{i} \in \Psi^{i}(s)$, then $\left(\alpha s_{1}^{i}+(1-\alpha) s_{2}^{i}\right) \in \Psi^{i}(s)$, where $0 \leq \alpha \leq 1$. Note that a strategy defined by $\alpha s^{i}$ consists of the flow pattern characterized by $s^{i}$, but instead of routing a flow of $f_{e}^{i}$ on an edge $e$, user $i$ now routes a flow of $\alpha f_{e}^{i}$ on $e$. As $s_{1}^{i}, s_{2}^{i}$ are best strategies of $i$ given $s^{-i}$, it follows that $c^{i}\left(s^{-i}, s_{1}^{i}\right)=c^{i}\left(s^{-i}, s_{2}^{i}\right)=c_{\min }^{i}\left(s^{-i}\right)$, where $c_{\min }^{i}\left(s^{-i}\right)$ denotes the cost of the minimum cost flow pattern of $i$ given the flow patterns $s^{-i}$ of all other users. We thus have to prove that this is also the cost of the strategy defined by $\left(\alpha s_{1}^{i}+(1-\alpha) s_{2}^{i}\right)$.

We show that, given $s^{-i}$, the cost of a flow $f_{e}^{i}$ of user $i$ on edge $e$ is a convex function. Denote by $n_{e}$ the number of users using $e$, and generate the ordering $f_{e, 1} \leq f_{e, 2} \leq \cdots \leq f_{e, j-1} \leq f_{e, j} \leq \cdots \leq f_{e, n_{e}}$ on the flow routed by the $n_{e}$ users on $e$ as defined by $s^{-i}$. Define $f_{e, 0}=0$. Denote by $W_{\left(f_{e, j}-f_{e, j-1}\right)}$ the total weight of the $n_{e}-j+1$ players using the flow fraction $\left[f_{e, j-1}, f_{e, j}\right]$. Clearly, $W_{f_{e, 1}} \geq W_{\left(f_{e, 2}-f_{e, 1}\right)} \geq \cdots \geq$ $W_{\left(f_{e, j}-f_{e, j-1}\right)} \geq \cdots \geq W_{\left(f_{e, n_{e}}-f_{e, n_{e}-1}\right)}$ (in case $f_{e, j-1}=f_{e, j}$ we define $W_{\left(f_{e, j}-f_{e, j-1}\right)}=W_{\left(f_{e, j-1}-f_{e, j-2}\right)}$ ). Now, assume that user $i$ wants to use edge $e$ as well. The cost of each possible fraction of $i$ 's flow on $e$ is described by Figure 6, and its total cost for routing an $f_{e}^{i}$-value flow on $e$ is described by Figure 7. 


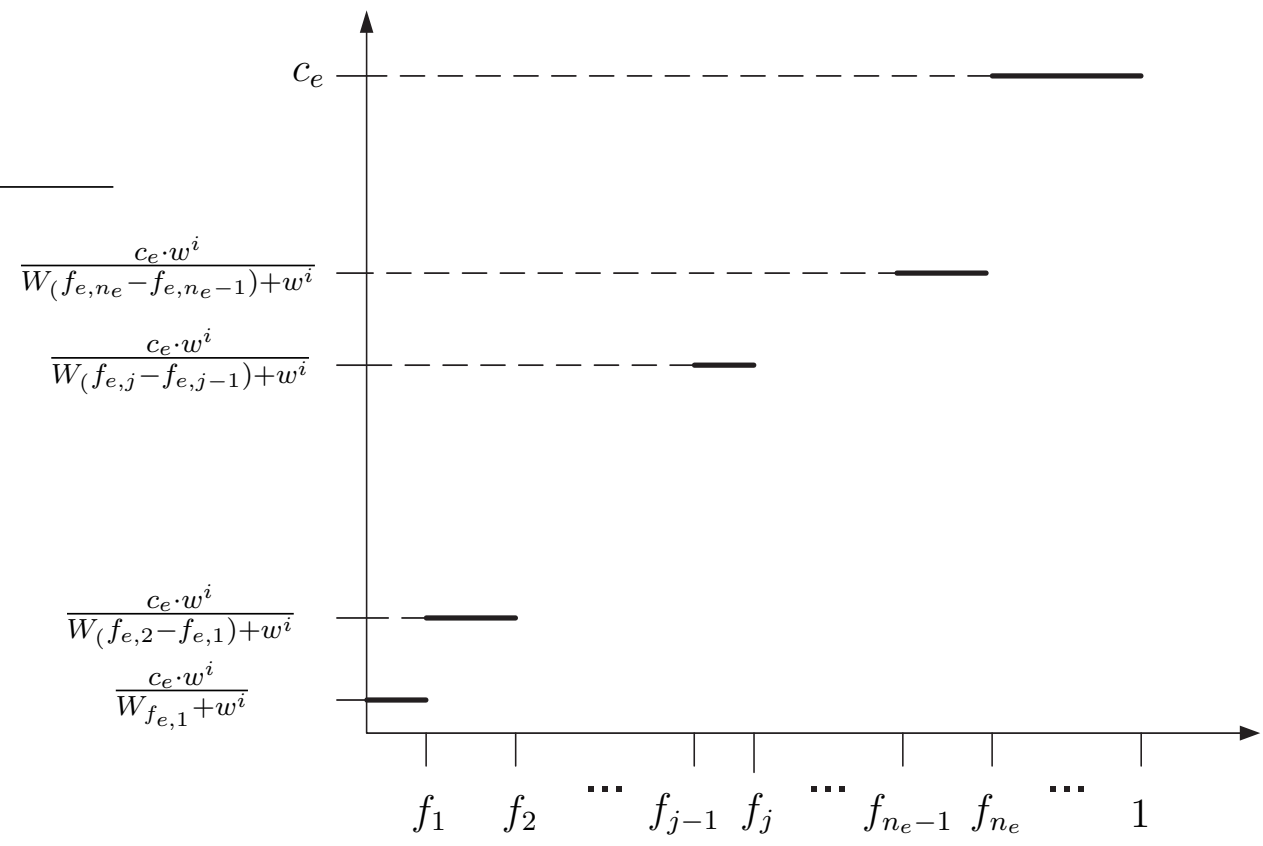

Fig. 6. Cost of each possible fraction of $i$ 's flow on edge $e$.

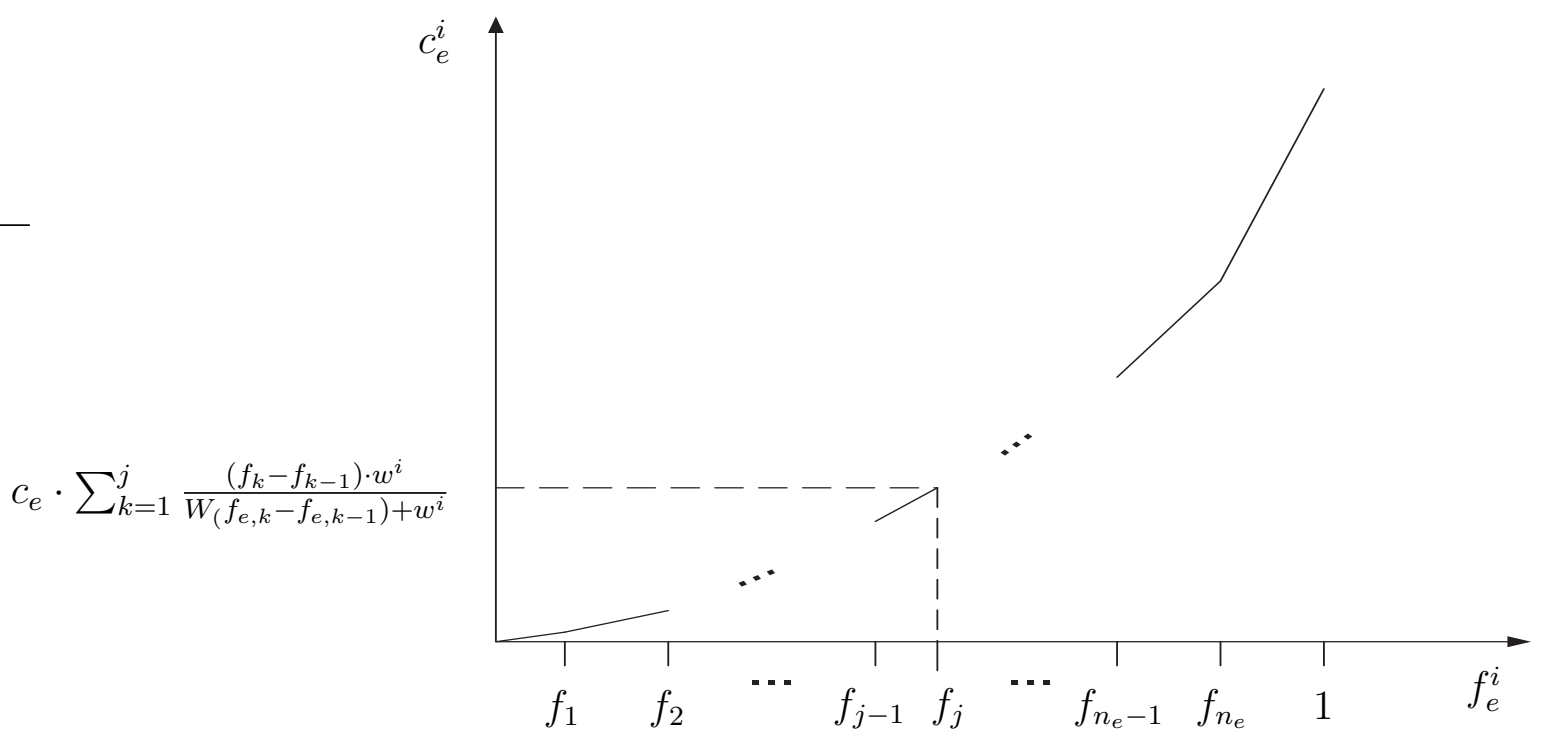

Fig. 7. Total cost of a flow of value $f_{e}^{i}$ routed by $i$ on edge $e$.

Clearly, the cost of user $i$ 's flow $f_{e}^{i}$ on edge $e$, denoted by $c_{e}^{i}\left(f_{e}^{-i}, f_{e}^{i}\right)$, is a convex function, as for each flow fraction $\left(f_{j+1}-f_{j}\right)$ the slope of the function is bigger than its slope for the previous fraction $\left(f_{j}-f_{j-1}\right)$. We denote by $\left(f_{e}^{i}\right)_{1},\left(f_{e}^{i}\right)_{2}$ the respective flows of $i$ on edge $e$ as defined by the strategies $s_{1}^{i}, s_{2}^{i} \in \Psi^{i}(s)$. Therefore, it holds that

$$
c_{e}^{i}\left(f_{e}^{-i}, \alpha\left(f_{e}^{i}\right)_{1}+(1-\alpha)\left(f_{e}^{i}\right)_{2}\right) \leq \alpha c_{e}^{i}\left(f_{e}^{-i},\left(f_{e}^{i}\right)_{1}\right)+(1-\alpha) c_{e}^{i}\left(f_{e}^{-i},\left(f_{e}^{i}\right)_{2}\right) .
$$

Thus, the same holds also for the total cost of $i$ with respect to strategy $\left(\alpha s_{1}^{i}+(1-\alpha) s_{2}^{i}\right)$, namely:

$$
\begin{aligned}
c^{i}\left(s^{-i}, \alpha s_{1}^{i}+(1-\alpha) s_{2}^{i}\right) & \leq \alpha c^{i}\left(s^{-i}, s_{1}^{i}\right)+(1-\alpha) c^{i}\left(s^{-i}, s_{2}^{i}\right) \\
& =c_{\min }^{i}\left(s^{-i}\right),
\end{aligned}
$$


which implies that $\left(\alpha s_{1}^{i}+(1-\alpha) s_{2}^{i}\right) \in \Psi^{i}(s)$, completing the proof of lemma 1.1.

Lemma 1.2: The correspondence $\Psi^{i}$ is upper hemicontinuous.

Proof: We consider a sequence $s_{r}$ in $S$ converging to $s$, and a sequence $s_{r}^{i}$ in $S^{i}$ converging to $s^{i}$, where $s_{r}^{i} \in \Psi^{i}\left(s_{r}\right)$ for every $r$. To show that $\Psi^{i}$ is upper hemicontinuous, we prove that $s^{i} \in \Psi^{i}(s)$.

We take $r$ to be sufficiently large, so that $s_{r}$ differs from $s_{r-1}$ by a routing change of an $\epsilon$-value flow. That is, $s_{r}$ is obtained from $s_{r-1}$ if user $j$ reroutes an $\epsilon$-value of its flow on path $p_{1}^{j}$ to another path $p_{2}^{j}$. According to the way we defined the users' costs on an edge, the change of $j$ 's flow pattern can only reduce the cost of an $\epsilon$-value of the flow on each edge in $p_{2}^{j}$, and increase the cost of an $\epsilon$-value of the flow on each edge in $p_{1}^{j}$, for all players using this $\epsilon$-value flow on the edges in $p_{2}^{j}$ and $p_{1}^{j}$ respectively.

Claim 2: An addition of an $\epsilon$-value flow to an edge $e$ can cause user $i$ to change the routing of no more than an $\epsilon$-value of its flow. Equivalently, the same holds for a deduction of an $\epsilon$-value flow from an edge $e$.

Proof: In case of the addition of an $\epsilon$-value flow to edge $e$, only the fractional cost of an $\epsilon$-value of flow on $e$ is reduced for user $i$, and all other flow costs remain the same. Therefore, any change in $i$ 's best reply involving a $\delta$-value of $i$ 's flow, where $\delta>\epsilon$, implies that the cost of a $(\delta-\epsilon)$-value of user $i$ 's flow could have been reduced prior to the flow addition on $e$.

Equivalently, the same holds in case of a deduction of an $\epsilon$-value flow from edge $e$. In this case, only the fractional cost of an $\epsilon$-value of flow on $e$ is increased.

Following the change of $j$ 's flow pattern, the most significant change in $s_{r}^{i}$ compared to $s_{r-1}^{i}$ would be if $i$ was to reroute a value of $2 \epsilon$ of its flow to different paths: an $\epsilon$-value rerouted from a set of paths, each containing an edge in $p_{2}^{j}$ (where its cost was increased); and an $\epsilon$-value rerouted to a set of paths, each containing another edge in $p_{2}^{j}$ (where its cost was reduced). Denoting the number of edges in $G$ by $|E|=m, i$ would therefore reroute no more than a $(2 m \cdot \epsilon)$-value of its flow.

We denote by $d(x, y)$ the distance between two vectors $x, y$ in an Euclidian space $\mathbb{R}^{k}$. In our context, a flow vector is in $S$. Thus, for every $\epsilon>0$, there is an index $R$ such that $r \geq R$ implies that $d\left(s_{r}^{i}, s^{i}\right)<\epsilon$ and $d\left(s_{r}, s\right)<\epsilon$, where $s_{r}^{i} \in \Psi^{i}\left(s_{r}\right)$. From the continuity property of $\Psi^{i}\left(s_{r}\right)$ shown above, it follows that $s^{i} \in \Psi^{i}(s)$.

We prove the existence of a Nash equilibrium using Kakutani's Fixed Point Theorem [17]:

Theorem 10 (Kakutani): If $T$ is a nonempty, compact, and convex subset of an Euclidian space, and $\Psi$ is an upper hemicontinuous, nonempty, and convex-valued correspondence from $T$ to $T$, then $\Psi$ has a fixed point, that is, there is an $x \in T$ such that $x \in \Psi(x)$.

We now finish the proof of Theorem 9.

Proof: We define a correspondence $\Psi$ from $S$ to $S$ by

$$
\Psi(s)=\Psi^{1}(s) \times \cdots \times \Psi^{n}(s) .
$$

As shown before, the set $S$ is a compact and convex subset of the Euclidian space. The correspondence $\Psi$ is upper hemicontinuous, nonempty, and convex-valued, since so is each $\Psi^{i}$, as shown in lemmas 1.1 and 1.2. Thus, by Kakutani's Fixed Point Theorem, there is a fixed point $s \in \Psi(s)$. It is easy to see that such a fixed point $s$ of $\Psi$ is a Nash equilibrium of $\Gamma$, as for each player $i$, its strategy prescribed by $s$ minimizes its cost given the strategies of the other players prescribed by $s$. 